\title{
Load Balancing Content-Based Publish/Subscribe Systems
}

\author{
ALEX KING YEUNG CHEUNG and HANS-ARNO JACOBSEN \\ University of Toronto
}

Distributed content-based publish/subscribe systems suffer from performance degradation and poor scalability caused by uneven load distributions typical in real-world applications. The reason for this shortcoming is the lack of a load balancing scheme. This article proposes a load balancing solution specifically tailored to the needs of content-based publish/subscribe systems that is distributed, dynamic, adaptive, transparent, and accommodates heterogeneity. The solution consists of three key contributions: a load balancing framework, a novel load estimation algorithm, and three offload strategies. A working prototype of our solution is built on an open-sourced contentbased publish/subscribe system and evaluated on PlanetLab, a cluster testbed, and in simulations. Real-life experiment results show that the proposed load balancing solution is efficient with less than $0.2 \%$ overhead; effective in distributing and balancing load originating from a single server to all available servers in the network; and capable of preventing overloads to preserve system stability, availability, and quality of service.

Categories and Subject Descriptors: C.2.3 [Computer-Communications Networks]: Network Operations-Network management; C.2.4 [Computer-Communications Networks]: Distributed Systems-Client/server; C.4 [Performance of Systems]: Measurement techniques

General Terms: Algorithms, Experimentation, Performance

Additional Key Words and Phrases: Publish/subscribe, content-based routing, load balancing, load estimation, subscriber migration, offload algorithms, PADRES, ToPSS

ACM Reference Format:

Cheung, A. K. Y. and Jacobsen, H.-A. 2010. Load balancing content-based publish/subscribe systems. ACM Trans. Comput. Syst. 28, 4, Article 9 (December 2010), 55 pages.

DOI: $10.1145 / 1880018.1880020$. http://doi.acm.org/10.1145/1880018.1880020.

This research is part of the PADRES project, which was supported in parts by the Ontario Centers of Excellence (OCE), the Canada Foundation for Innovation (CFI), the Ontario Innovation Trust (OIT), and the Natural Sciences and Engineering Research Council of Canada (NSERC). Industrial sponsorship was provided by CA Technologies, Inc., Cybermation, Inc., and Sun Microsystems for parts of this research.

Authors' address: A. K. Y. Cheung and H.-A. Jacobsen, Department of Electrical and Computer Engineering, University of Toronto, 10 King's College Road, Toronto, Ontario, M5S 3G4, Canada; email: \{cheung; jacobsen\}@eecg.utoronto.ca.

Permission to make digital or hard copies part or all of this work for personal or classroom use is granted without fee provided that copies are not made or distributed for profit or commercial advantage and that copies show this notice on the first page or initial screen of a display along with the full citation. Copyrights for components of this work owned by others than ACM must be honored. Abstracting with credit is permitted. To copy otherwise, to republish, to post on servers, to redistribute to lists, or to use any component of this work in other works requires prior specific permission and/or a fee. Permissions may be requested from Publications Dept., ACM, Inc., 2 Penn Plaza, Suite 701, New York, NY 10121-0701 USA, fax +1 (212) 869-0481, or permissions@acm.org. (c) 2010 ACM 0734-2071/2010/12-ART9 $\$ 10.00$ DOI: $10.1145 / 1880018.1880020$ http://doi.acm.org/10.1145/1880018.1880020. 


\section{INTRODUCTION}

Content-based publish/subscribe is widely used in large-scale distributed applications because it allows processes to communicate asynchronously in a loosely and decoupled manner. This property gives systems higher modularity and reuse as well as easier maintainability. In publish/subscribe, loose-coupling is achieved by simply having producers publish information into the network without knowing the identity, location, and number of subscribers. Likewise, consumers subscribe to specific information without knowing the identity, location, and number of publishers. Topic-based publish/subscribe systems [Castro et al. 2002; Chen et al. 2010; Oki et al. 1993] too offer loose-coupling benefits, but lack the language expressiveness, finer grained in-network filtering, and complex event processing capabilities of content-based publish/subscribe systems [Banavar et al. 1999; Carzaniga et al. 2001; Fabret et al. 2001; Li and Jacobsen 2005; Li et al. 2008a; Pietzuch and Bacon 2002]. The added flexibility of content-based filtering is already realized in commercial systems such as GooPS [Reumann 2009], Google's publish/subscribe system that integrates its web applications; SuperMontage [Tibco 2001], Tibco's publish/subscribe distribution network for Nasdaq's quote- and order-processing system; GDSN (Global Data Synchronization Network) [GS1 2008], a global publish/subscribe network that allows suppliers and retailers to exchange timely and accurate supply chain data; and in other applications such as multiplayer online games [Bharambe et al. 2002], decentralized business process execution [IBM et al. 2007; Li et al. 2010; Muthusamy et al. 2007; Schuler et al. 2001], workflow management [Cugola et al. 2001], business activity monitoring [Fawcett and Provost 1999], network and systems monitoring [Mukherjee et al. 1994; Vitria 2008], automated service composition [Hu et al. 2008], RSS filtering [Petrovic et al. 2005; Rose et al. 2007], resource discovery [Yan et al. 2009] and the Enterprise Service Bus of Service Oriented Architecture infrastructures [IBM 2008; Microsoft 2008].

In any distributed publish/subscribe system, brokers located at different geographical areas may suffer from uneven load distribution due to different population densities, interests, and usage patterns of end-users. A motivating application scenario is a supermarket supply chain with RFID tracked goods in GDSN, as modeled by the SPECjms2007 workload [Sachs et al. 2009]. Figure 1 illustrates the application scenario with one possible design of the publish/subscribe cloud. SPECjms2007 models seven interactions between the company headquarters (HQ), supermarkets (SM), distribution centers (DC), and suppliers (SP). The interactions involve both multicast and point-to-point communications, both of which are supported by the publish/subscribe paradigm. Here, we simply present the interaction involving order/shipment handling between suppliers and distribution centers to demonstrate our point.

(1) A DC sends a call for offers to all SPs that supply the types of goods that need to be ordered.

(2) SPs that can deliver the goods send offers to the DC.

(3) Based on the offers, the DC selects an SP and sends a purchase order to it. 


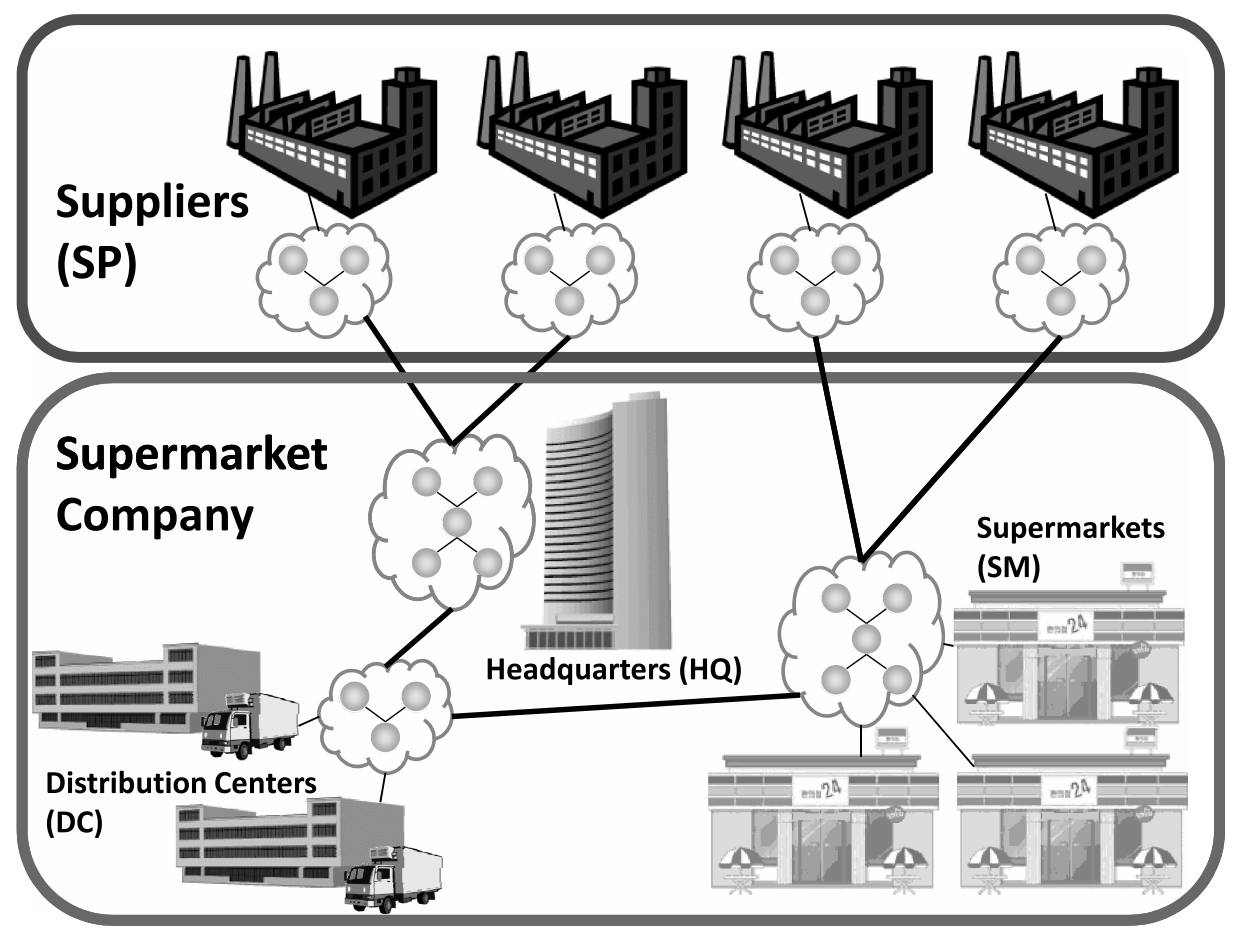

Fig. 1. SPECjms2007 workload scenario with an example publish/subscribe cloud deployment.

(4) The SP sends a confirmation to the DC and an invoice to the HQ. It then ships the ordered goods.

(5) The shipment arrives at the DC and is registered by RFID readers upon entering the DC's warehouse.

(6) The DC sends a delivery confirmation to the SP.

(7) The DC sends transaction statistics to the HQ.

Just from this one single interaction pattern, it is possible that brokers at any one of SP, DC, or HQ can be overloaded due to unexpected high order rates (such as the first business day after a long weekend), heterogeneous servers at different sites, reduced server resources due to failures, network congestion, high CPU and/or bandwidth usage from other processes on the same server, etc. Focusing on publish/subscribe alone, a broker can be overloaded in two ways. First, the broker can be overloaded if the incoming message rate into the broker exceeds the processing/matching rate of the matching engine. This effect is exacerbated if the number of subscribers is large because the matching rate is inversely proportional to the number of subscriptions in the matching engine [Fabret et al. 2001]. Second, overload can also occur if the output transmission rate exceeds the total available output bandwidth. In both cases, queues accumulate with increasingly more messages waiting to be processed, resulting in increasingly higher processing and delivery delays. Worse yet, 
the broker may crash when it runs out of memory from queueing too many messages.

The matching rate and both the incoming and outgoing message rates determine the load of a broker. In turn, these factors depend on the number and nature of subscriptions that the broker services. Thus, load balancing is possible by offloading subscriptions from higher loaded to lesser loaded brokers. However, the use of a generic load balancing scheme with no publish/subscribe context is not a viable option. Our experiments on PlanetLab and a cluster testbed proves this point by showing that random selection of subscriptions makes the load balancer unstable and jeopardizes the entire system even if the number of subscriptions offloaded is meaningfully calculated.

Hence, we develop a new load balancing algorithm that distributes load by offloading strategically chosen subscriptions from heavily loaded brokers to less loaded brokers. Our contributions to support this idea include (1) a framework that facilitates load balancing by isolating subscribers to the edge brokers in the network and a set of protocols for triggering and mediating load balancing sessions; (2) a novel load estimation algorithm that profiles subscription load using bit vectors; (3) offload algorithms that load balance on multiple performance metric of a broker by selecting the appropriate subscriptions to offload based on their profiled load characteristics; and (4) experimental results that demonstrate the performance and effectiveness of our load balancing solution. Additionally, we have significantly extended our previous work [Cheung and Jacobsen 2006] with new algorithms that improve performance and further evaluations on real testbeds. Specifically, the new extensions include:

(1) A naive offload algorithm to serve as a comparison against a generic load balancing scheme and to highlight the need and strength of our solution.

(2) A more efficient load exchange protocol called Adaptive PIE that leverages the infrastructure's publish/subscribe operations to further reduce message overhead.

(3) Techniques to accurately model multiple performance metrics on real deployments, namely the input utilization ratio, matching delay, and output utilization ratio.

(4) A working prototype of all presented ideas in this article is built on the open source PADRES [Cheung and Jacobsen 2006; Li and Jacobsen 2005; Li et al. 2007; MSRG 2008] distributed content-based publish/subscribe system.

(5) Results from live experimentation on PlanetLab, which puts our ideas under harsh real-world Internet-scale conditions, and a cluster testbed that mimics running our approach under local area network and data-center conditions.

(6) Comparison and discussion of simulation and real-world results on heterogeneous and homogeneous environments using the proposed and naive offload algorithms.

The rest of this article is organized as follows. Section 2 provides background on publish/subscribe and discusses related work. Section 3 presents 
the load balancing framework. Section 4 describes the load estimation algorithm. Section 5 describes the three offload algorithms as well as the naive offload algorithm. Section 6 presents and compares both real and simulated experiment results. Finally, Section 7 concludes this article.

\section{BACKGROUND AND RELATED WORK}

\subsection{Content-Based Publish/Subscribe Language}

In publish/subscribe, clients that send publication messages into the system are referred to as publishers, while those that only receive messages are called subscribers. Publishers issue publications/events ${ }^{1}$ with information arranged in attribute key-value pairs. An example stock publication with eight key-value pairs is represented as:

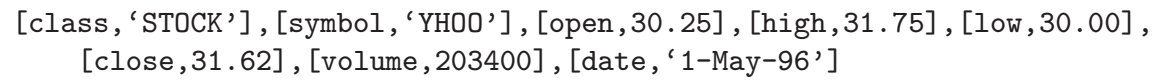

The key class denotes the topic of the publication; symbol denotes the symbol of the stock and carries a string value of YHOO. The stock's open, high, low, close, and volume values are numeric and hence unquoted.

Subscribers issue subscriptions to specify the type of publications they want to receive. In topic-based publish/subscribe, subscribers can only specify the topic of interest in their subscriptions. In content-based publish/subscribe, subscribers can specify more complex constraints in addition to just specifying the topic of interest. Depending on the implementation, subscription filters in content-based publish/subscribe can be based on attributes (which this article focuses on), on path expressions through the use of XML [Altinel and Franklin 2000; Hou and Jacobsen 2006; Li et al. 2008a; Pereira et al. 2001], or on graph expressions [Petrovic et al. 2005]. Attribute-based predicates consist of an operator-value pair to specify the filtering conditions on each attribute. Some examples of operators used for string-type attributes include equal, prefix, suffix, and contains comparators, denoted as eq, str-prefix, str-suffix, and str-contains, respectively. For attributes containing numeric values, one can use the $=,>,<,>=$, and $<=$ operators. For example, a subscription for publications regarding the $Y H O O$ stock whenever its volume is greater than 300,000 is indicated as follows:

[class, eq, 'STOCK'] , [symbol, eq, 'YHOO'] , [volume ,> , 300000] .

The space of interest defined by a subscription's filtering conditions is called subscription space. A broker's covering subscription set (CSS) refers to the set of most general subscriptions whose subscription space is not covered by any other subscription managed by this broker. For example, a broker with the set of subscriptions shown in Figure (2)a has a CSS identified by the bolded subscriptions.

\footnotetext{
${ }^{1}$ In the publish/subscribe literature, the terms publications and events are often used synonymously.
}

ACM Transactions on Computer Systems, Vol. 28, No. 4, Article 9, Pub. date: December 2010. 


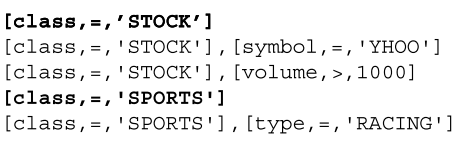

(a) Subscriptions

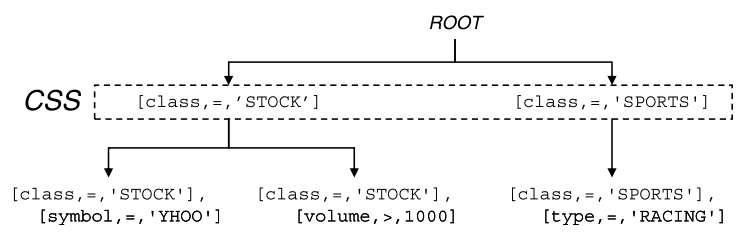

(b) Poset

Fig. 2. Example of the poset data structure.

For more efficient retrieval of a broker's CSS, the partially-ordered set (poset) [Carzaniga et al. 2001] is used to maintain subscription relationships. The poset is a directed acyclic graph where each unique subscription is represented as a node in the graph as shown in Figure 2(b). Nodes can have parent and children nodes where parent nodes have a subscription space that is a superset of its children nodes, while subscriptions with intersection or empty relationships will appear as siblings. As shown, the CSS is readily available as the immediate children nodes under the imaginary ROOT node.

Subscribers issue unsubscription messages whenever they disconnect from the system. Unsubscription messages do not contain any predicates, but an identifier that correlates to the subscription to be removed from the system.

In some content-based publish/subscribe systems, such as SIENA [Carzaniga et al. 2001], REBECA [Mühl 2001], and PADRES [Fidler et al. 2005; Li et al. 2008b; MSRG 2008], advertisements are sent by publishers prior to sending the first publication message. For example, a publisher of the publication previously outlined would have an advertisement such as this:

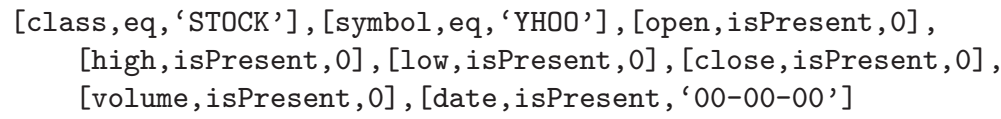

It may not always be possible to know the exact range of an attribute's value before publishing. Hence, the isPresent operator is used to denote that the attribute merely exists with a string or numeric type. Advertisements are flooded throughout the network so that subscriptions do not have to be flooded but instead travel along the reverse path of matching advertisements. In publish/subscribe systems without advertisements, subscriptions can be flooded. It is also conceivable that publications are flooded, which under normal operating assumptions is the least desirable approach. For many publish/subscribe application scenarios, it is assumed that advertisements constitute the least number of messages, followed by subscriptions, and finally by publications constituting the largest number of messages. For example, newspaper agencies rarely change, but the number of news readers subscribing and unsubscribing from a news service is comparatively higher. For scenarios where the above assumption about message type frequencies does not hold, protocols exist that can handle these situations [Muthusamy et al. 2005]. Unadvertisement messages are used by the publisher whenever it disconnects from the system. Our load balancing solution is applicable regardless of whether advertisements and unadvertisements are used by the publish/subscribe system. 


\subsection{Publish/Subscribe Architectures}

Two main classes of distributed content-based publish/subscribe systems exists today: filter-based and multicast-based. In the filter-based approach, subscriptions are propagated into the network to establish paths that guide publications to subscribers. Each publication is matched at every broker along the overlay to get forwarded towards neighbors with matching subscriptions. Consequently, the farther the publication travels, the higher is the delivery delay. The overlay of filter-based approaches may consist of either a set of dedicated brokers [Banavar et al. 1999; Carzaniga et al. 2001; Chen and Schwan 2005; Cugola et al. 2001; Mühl 2001; Li et al. 2008b; MSRG 2008; Pallickara and Fox 2003; Pietzuch and Bacon 2002; Segall and Arnold 1997] or purely clients [Aekaterinidis and Triantafillou 2006; Baldoni et al. 2005; Castelli et al. 2008; Chand and Felber 2005; Gupta et al. 2004; Patel et al. 2009; Tam et al. 2003; Terpstra et al. 2003; Triantafillou and Aekaterinidis 2004; Voulgaris et al. 2006; Zhang et al. 2005; Zhu and $\mathrm{Hu}$ 2007]. In the multicast-based approach [Adler et al. 2001; Casalicchio and Morabito 2007; Opyrchal et al. 2000; Riabov et al. 2002, 2003; Wong et al. 2000], subscribers with similar interests are clustered into the same multicast group. Each publication is matched once to determine the matching multicast group(s) to which the message should be multicasted, broadcasted, or unicasted. As a result, matching and transmission of a publication message happens at most once, thus incurring minimal delivery delay. However, compared to the filter-based approach, subscribers in a multicast group may receive unwanted publications because subscribers with even slightly different interests may still be assigned to the same group. One possible solution to this problem is the introduction of filter-based functionality within each multicast group [Cao and Singh 2004].

Even though our work is targeted at filter-based publish/subscribe systems consisting of an overlay of dedicated brokers, it is also applicable to topic-based publish/subscribe systems. ${ }^{2}$ This is because brokers in both systems overload due to the same patterns, and the relationship among any two subscriptions in a topic-based system is a subset of those in a content-based system. (Topicbased can only have equal and empty relationships, whereas content-based can have equal, empty, subset, superset, and intersect relationships.) Additionally, parts of our load balancing framework can be leveraged to benefit filter-based approaches consisting of only clients as well as multicast-based approaches. For example, in P2P approaches such as Baldoni et al. [2007], the idea of subscribing to load information from a partial set of nodes as used in our load exchange protocol (later referred to as PIE) can be leveraged to allow nodes to exchange load information while remaining loosely coupled with each other. In multicast-based approaches, (1) PIE and Adaptive PIE can be leveraged to give clustering algorithms awareness of server loads. (2) PRESS can also be used to accurately estimating the load of a set of subscriptions and avoid clustering too many subscriptions into a single group/server. (3) Our offload algorithms can be utilized to temporarily stop overloads before re-clustering takes place.

${ }^{2}$ Except the Adaptive PIE extension in Section 3.3.4. 


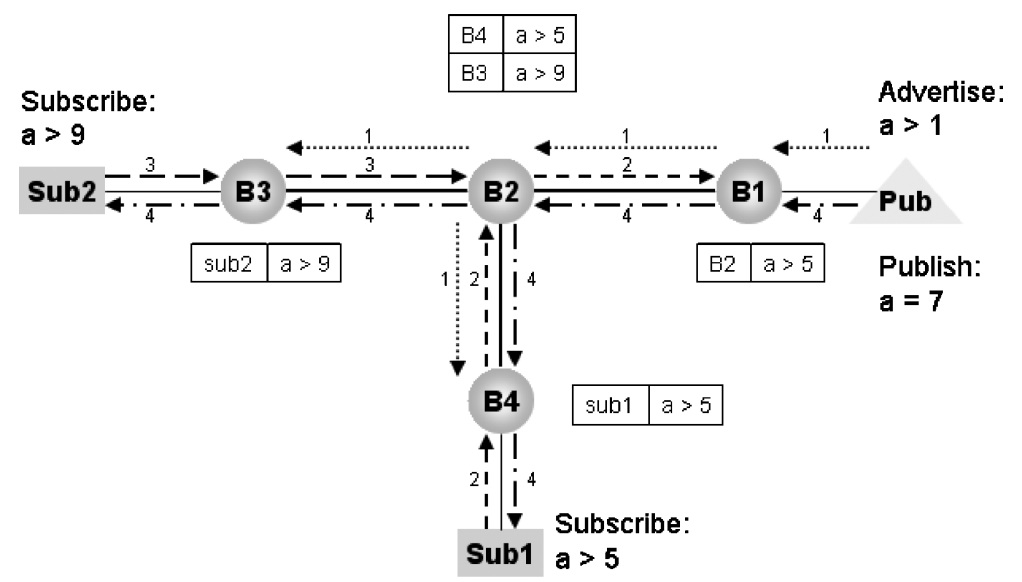

Fig. 3. Example of content-based publish/subscribe routing.

\subsection{Filter-Based Publish/Subscribe Routing}

Figure 3 demonstrates an example of how advertisements, subscriptions, and publications are routed. In the first step, the publisher (represented by a triangle) advertises $a>1$ to broker $B 1$. As shown by the arrows labeled with 1 , the advertisement is flooded to all brokers in the network. Subscribers never receive advertisements because advertisements are control messages and subscribers only receive publication messages that match their subscription filter. In Step (2), subscriber sub1 issues its subscription a>5 to broker $B 4$. Since sub1's subscription's space is common with the publisher's advertisement space, sub1's subscription is forwarded along the reverse path of the advertisement, as shown by the arrows labeled with 2 . Notice that the publisher does not receive the subscription because subscriptions are control traffic within the publish/subscribe system and publishers never receive messages. In Step (3), subscriber $s u b 2$ issues its subscription a $>9$ to broker B3. Similar to sub1's subscription, sub2's subscription travels in the reverse path of the publisher's advertisement since it matches with a subset of the advertisement space. However, once the subscription reaches broker $B 2$, the subscription is purposely not forwarded to broker $B 1$ because sub2's subscription is covered by sub1's. In other words, since $B 2$ is already receiving publication messages that matches sub2's subscription space due to sub1's subscription, it is not necessary to forward sub2's subscription to B1. As illustrated in the figure, publications sent by the publisher in Step (4) will get delivered to both subscribers according to the brokers' publication routing tables shown beside each broker (with the left and right columns representing the next-hop and subscription filter, respectively). When subscriber sub1 unsubscribes from broker $B 4$ in the future, the unsubscription will traverse along the same path as sub1's original subscription up to broker $B 1$. When the unsubscription reaches broker B2, B2 forwards sub2's subscription to $B 1$ before sub1's unsubscription to avoid interrupting sub2's event delivery. 


\subsection{Load Balancing}

Load balancing has been a widely explored research topic for the past two decades since the introduction of parallel and distributed computing. The goal of all load balancing solutions is to evenly distribute workload to all available resources. Load balancing solutions can be found in four software layers: network [Cardellini et al. 1999a,b; Dias et al. 1996], operating system [Jul et al. 1988; Litzkow 1987; Shirriff 1997; Zajcew et al. 1993], middleware [Aleksy et al. 2001; Barth et al. 1999; Ho and Leong 2000; Lindermeier 2000; Tatbul et al. 2007], and application [Berman and Wolski 1996; Othman et al. 2003]. The layer in which a load balancing strategy is implemented depends on which layer contains the logic to make effective load balancing decisions. For example, it would be ineffective to use a random DNS redirection strategy in the network layer or process migration in the OS layer to load balance a contentbased publish/subscribe system that resides at the middleware layer. This is because these approaches cannot identify the relationship (whether it be intersecting, superset, subset, equal, or empty) between subscriptions nor estimate the load of a subscription imposed onto a broker already servicing an arbitrary set of subscriptions. We demonstrate this claim through a naive offload algorithm that randomly picks subscriptions to offload. Experiments show that the naive algorithm does lead to an unstable system on PlanetLab and the cluster testbed.

\subsection{Load Balancing in Content-Based Publish/Subscribe}

To the best of our knowledge, Cheung and Jacobsen [2006] was among the first to address load balancing in content-based publish/subscribe systems although distributed content-based publish/subscribe systems have been widely studied. The following are various related approaches that propose load balancing techniques in other publish/subscribe approaches.

Meghdoot [Gupta et al. 2004] is a peer-to-peer content-based publish/ subscribe system based on a distributed hash table that distributes load by replicating or splitting the locally heaviest loaded peer in half to share the responsibility of subscription management or event propagation. Such partitioning and replication schemes are common load balancing techniques used in structured P2P publish/subscribe systems [Aekaterinidis and Triantafillou 2006; Zhu and $\mathrm{Hu}$ 2007]. In general, their load sharing algorithm is only invoked upon new peers joining the system and peers are assumed to be homogeneous.

Chen and Schwan [2005] proposed a dynamic overlay reconstruction algorithm called Opportunistic Overlay that reduces end-to-end delivery delay and also performs load distribution on the CPU utilization as a secondary requirement. Load balancing is triggered only when a client finds another broker that is closer than its home broker. It is possible that subscriber migrations may overload a broker if the load requirements of the migrated subscription exceed the load-accepting broker's processing capacity.

Subscription clustering is another technique to achieve load balancing in content-based publish/subscribe systems [Casalicchio and Morabito 2007; 


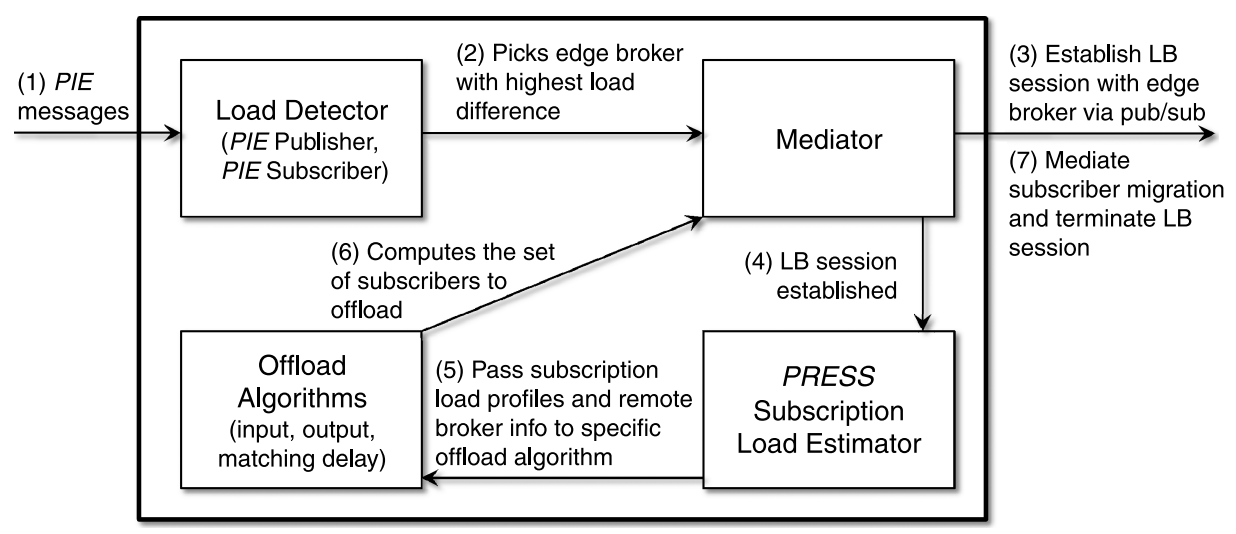

Fig. 4. Components of the load balancer.

Riabov et al. 2002, 2003; Wong et al. 2000]. Load balancing is done by partitioning the set of subscriptions into a predefined number of servers or groups. The partitioning clusters subscriptions of highest common interest together to minimize the total amount of network traffic. However, architecturally, this technique is not applicable to filter-based but only to multicast-based publish/subscribe systems.

Our work differs from the prior three solutions by proposing a distributed load balancing algorithm for content-based publish/subscribe systems that accounts for heterogeneous brokers and subscribers, and distributes load evenly onto all resources in the system even without new clients joining. We also present a detailed subscriber migration protocol that enforces end-user transparency and best-effort delivery to minimize message loss.

\section{LOAD BALANCING FRAMEWORK}

Before we describe each component of our load balancing approach in great detail, we first give a high-level overview to show how components interact with each other. Each broker in the system runs an instance of the load balancer whose components are shown in Figure 4. In general, the load balancer detects overload and load imbalance, upon which specific subscriptions are offloaded to a broker with more available resources. Specifically, the load balancer consists of the Load Detector and Mediator as described in this section, PRESS Subscription Load Estimator as described in Section 4, and Offload Algorithms as described in Section 5. In the following, we will use the numbering in this figure as a guide to explain the control flow of a typical load balancing session. (1) The Load Detector detects if its own broker is overloaded or the load of its own broker exceeds another broker by monitoring a number of performance metrics. (2) If overload or load imbalance is detected, then the detector tells the Mediator (3) to establish a load balancing session between its own broker, namely offloading broker (broker with the higher load doing the offloading) and 


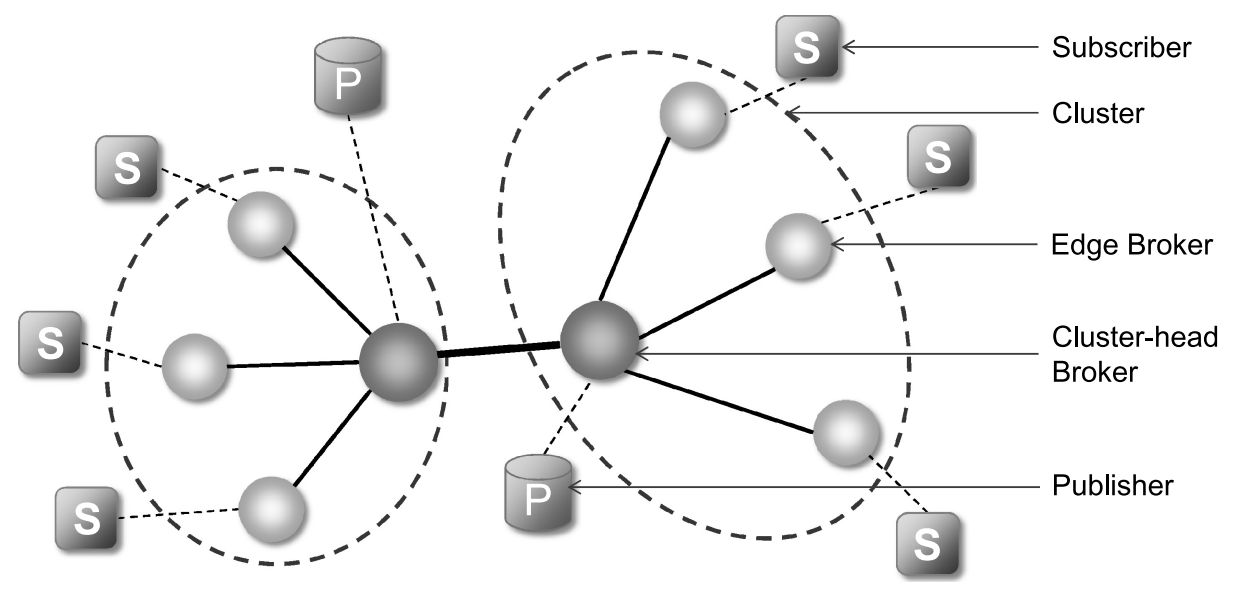

Fig. 5. PEER architecture.

a load-accepting broker (broker accepting load from the offloading broker). (4) Once the load balancing session is established, (5) the offloading broker collects publication delivery statistics for each of its subscription through PRESS and passes this information to one of the three offload algorithms. (6) Each offload algorithm is specifically designed to reduce the load on one of the performance metrics, which means the performance metric that is overloaded or needs balancing determines which offload algorithm to invoke. (6) The offload algorithm strategically selects the set of subscriptions to offload and passes this set to (7) the Mediator to coordinate the subscriber migration process. The load balancing session is over once the subscriber migration is complete. The following sections will describe the load balancing framework and the operations of each component in much greater detail.

\subsection{Structuring the Overlay into Clusters}

The Publish/subscribe Efficient Event Routing (PEER) framework organizes brokers into a hierarchical structure as shown in Figure 5. PEER is motivated by the architecture adopted by Google's distributed publish/subscribe system, GooPS [Reumann 2009], where each data center consists of a cluster of publish/subscribe brokers, and data centers in different geographical areas are connected by dedicated network links. Brokers with only one neighbor are referred to as edge brokers, while brokers with more than one neighboring broker are referred to as cluster-head brokers. A cluster-head broker with its connected set of edge brokers, if any, forms a cluster. Brokers within a cluster are assumed to be closer to each other in network proximity than brokers in other clusters. Publishers are serviced by cluster-head brokers, while subscribers are serviced by edge brokers. PEER supports the notion of a multi-level hierarchical network with clusters of smaller clusters, but in this article we will limit our scope to one level for the sake of simplicity. 
PEER is designed with five goals in mind. First, PEER allows the load balancing scheme to control the load of edge brokers simply by moving subscriptions because edge brokers have no publishers and no broker-to-broker through-traffic to route. Second, higher dissemination efficiency is achieved by having cluster-heads forward publication messages to all matching clusters almost simultaneously because cluster-heads have negligible processing delays since they do not service any subscribers. Third, cluster-head brokers may be load balanced by moving publishers and inter-broker subscriptions. Fourth, PEER's organization of brokers into clusters allows for two levels of load balancing: local-level (referred to as local load balancing) where edge brokers within the same cluster load balance with each other; and global-level (referred to as global load balancing) where edge brokers from two different clusters load balance with each other. Edge brokers only need to exchange load information with edge brokers in the same cluster, and neighboring clusters can exchange aggregated load information about their own edge brokers. Fifth, local load balancing preserves subscriber locality by keeping subscribers within their original cluster, assuming that subscribers connect to the closest broker in the first place. On the other hand, global load balancing trades off locality for a better balanced system by migrating subscribers between edge brokers in neighboring clusters.

\subsection{Messaging Framework}

All coordination messaging of the load balancing framework is done via publish/sub-scribe. Such messages are referred to as control messages or control publications as they are not observable to the end user unless explicitly subscribed upon. There are two primary reasons why publish/subscribe is used for control messaging. One, this approach unifies the messaging protocols of all components within the publish/subscribe system, thereby assuring ease of system maintainability. Second, the publish/subscribe paradigm directly meets the needs of the load balancing algorithm. For example, as part of the detection algorithm described in the next section, an edge broker can multicast a message to all other brokers within the cluster by a simple publish operation without having to worry about new or removed edge brokers in the cluster and manage connectivity with them.

In this work, we put three unique message attributes into use. First, control messages have higher priority than normal publication traffic so that the response time of the load balancer is not affected by the load of the broker. Second, the class of control messages demonstrated in Section 3.3.2 have a Time-To-Live (TTL) field that limits the hop count of the message to neighboring clusters. Third, the payload of a message is used to carry data that does not affect the routing of the message. This provides the benefit of reduced matching time at each broker.

\subsection{Load Detection Framework}

In order for brokers to know when and which brokers are available for load balancing, they have to exchange load information with each other. That way, a 
detection algorithm can trigger load balancing whenever it detects an overload or a wide load difference with another broker.

3.3.1 Broker Performance Metrics. Our performance metrics assume a general broker architecture consisting of an input queue to buffer incoming messages to the broker's matching engine; a matching engine which takes a message from the input queue, performs the matching (i.e., publications against subscriptions), generates and puts zero or more messages to route into the output queue; and at least one output queue to buffer messages from the matching engine to get transmitted to the next hop. Figure 10 shows an example of such a broker architecture.

The load of a broker is captured by three performance metrics: input utilization ratio, average matching delay per message, and output utilization ratio. Input utilization ratio $\left(I_{r}\right)$ captures the broker's processing or CPU utilization as well as the amount of time that incoming messages wait at the broker's input queue. $I_{r}$ is defined by the following formula, with $i_{r}$ representing the incoming publication rate in $\mathrm{msg} / \mathrm{s}$, and $m_{r}$ representing the maximum matching rate also in $\mathrm{msg} / \mathrm{s}$ :

$$
I_{r}=\frac{i_{r}}{m_{r}} .
$$

Maximum match rate, $m_{r}$, is calculated by taking the inverse of the average matching delay per message. $I_{r}$ can have any value greater than or equal to 0 . A value of 1.0 or greater for $I_{r}$ signifies that the broker is input overloaded.

Matching delay is defined as the time spent by the matching engine between taking a message as input and producing zero or more messages as output. The average matching delay metric is important because it captures the average amount of processing time each message undergoes when processed by a broker.

The output utilization ratio $\left(O_{r}\right)$ captures the broker's output bandwidth utilization and the amount of time messages spend waiting in the output queue before being sent off. $O_{r}$ is defined by the following formula with $o_{u}$ representing the output bandwidth usage in bytes per second, and $o_{t}$ representing the total amount of output bandwidth with the same units:

$$
O_{r}=\frac{o_{u}}{o_{t}} \text {. }
$$

Expanding on $o_{u}$ and $o_{t}$ will yield the following equation for $O_{r}$ :

$$
O_{r}=\left(\frac{t_{\text {busy }}}{t_{\text {window }}}\right)\left(\frac{b_{r x}}{b_{t x}}\right) \text {. }
$$

In Eq. (3), $t_{\text {window }}$ is the monitoring time window, and $t_{b u s y}$ is the amount of time spent sending messages within $t_{\text {window }}$. The fraction of these two variables yields a value range between 0 and 1 , where a value of 0 signifies that the output bandwidth is not used and 1 signifies that the resource is fully consumed. As for the last two variables, $b_{r x}$ represents the messages (in bytes) put into the output queue in time window $t_{\text {window }}$, and $b_{t x}$ represents the messages (in bytes) removed from the output queue and sent successfully in time window 
$t_{\text {window }} \cdot{ }^{3}$ The fraction of these two variables yields a value range greater than or equal to 1 , where a value greater than 1 signifies overload. If no messages are transmitted in the time window, then this fraction defaults to a value of 0 . At times when there is no overload, the left fraction indicates the bandwidth utilization while the right fraction yields a neutral value of 1 . At times of overload, the left fraction maximizes to a neutral value of 1 while the right fraction indicates the magnitude of overload by yielding a value greater than 1 .

3.3.2 Protocol for Exchanging Load Information. Publish/subscribe Information Exchange (PIE) is a distributed hierarchical protocol for exchanging load information between brokers using publish/subscribe primitives. Brokers publish PIE messages intermittently to let other brokers in the federation know of their existence, availability for load balancing, and load levels of each performance metric. As the next section describes, the detection algorithm uses information gathered from these PIE messages to determine whether and with which broker to engage in load balancing. PIE, as well as other load balancing control messages described in later sections, has a higher routing priority than normal publish/subscribe traffic so that their delivery is not affected by the broker's load. A PIE message contains five attributes:

(1) The broker's three performance metrics (as outlined in the previous section).

(2) Load balancing states, which can be one of $O K, B U S Y, N / A$, or STABILIZING.

(3) The set of edge brokers that this broker is currently balanced with (more on this in Section 3.4.1).

(4) The identifier of the cluster to which the broker belongs.

(5) The broker's unique identifier.

PEER's hierarchical structuring naturally allows for local and global PIE messages. Local PIE messages are published and subscribed by edge brokers within the same cluster to enable local load balancing. An example of a broker subscribing to local PIE messages within cluster ID "C01" is:

$$
\text { [class, =, 'LOCAL_PIE'], [cluster, =, 'C01'] . }
$$

Global PIE messages are published and subscribed by cluster-head brokers to enable global load balancing. They only propagate one cluster-hop away as enforced by a TTL of 1 and contain averaged load information from their cluster's local PIE messages. Cluster-head brokers without any edge brokers (such as a cluster-head of many cluster-heads in a multi-level hierarchical arrangement) simply forward global PIE messages one extra hop to all of their neighbors.

PIE messages are generated at a frequency defined by a parameter called local PIE publishing period (default is $30 \mathrm{~s}$ ) for the local-level and global PIE publishing period (default is $60 \mathrm{~s}$ ) for the global-level. According to our micro experiments in Cheung [2006], a low value will improve data liveliness, which

${ }^{3}$ With respect to the implementation, the byte count is taken by serializing the Java message object.

ACM Transactions on Computer Systems, Vol. 28, No. 4, Article 9, Pub. date: December 2010. 
in turn improves load balancing response time. However, frequent publishing of PIE messages increases overhead. Moreover, a low value may yield fluctuating load values due to temporary load spikes, which triggers unnecessary load balancing. Therefore, the publishing period should be set long enough to better capture the average load of a broker in the presence of load spikes. The global PIE publishing period should be set to a value that is equal to or higher than the local PIE publishing period because the data published in global PIE messages is aggregated from the local PIE messages received by the cluster-head brokers. If the global PIE publishing period is set lower than the local PIE publishing period, then global PIE messages published in between local PIE publishing period will report the same data, which leads to unnecessary overhead. For best load balancing response time, the global PIE publishing period should be set equal to or just above the local PIE publishing period. In summary, both parameters control the tradeoff between load balancing response time and overhead.

An additional constraint is that PIE messages are only published when one of the utilization ratios or the matching delay differs by a threshold from the previously published corresponding value. The purpose of this is to avoid publishing redundant information which wastes system resources. The thresholds are defined by the parameters PIE ratio threshold and PIE delay threshold, respectively. Both parameters default to 0.025 . Ideally, both thresholds should be set high enough to be effective in filtering out PIE messages that report similar data. However, too high of a threshold can increase the staleness of PIE information, which can hurt the load balancing response time. Given that PIE messages introduce very low overhead according to our experiments, both of these threshold values can be set conservatively. In short, this parameter controls the tradeoff between overhead and load balancing response time.

3.3.3 Detection Algorithm. Detection allows a broker/cluster to monitor its current resource usage and also compare it with other edge brokers/clusters so that a broker/cluster can invoke load balancing if necessary. Detection runs periodically at a broker/cluster only if it has a status of $O K, N / A$, and STABILIZING. An $O K$ status means that the broker is available for load balancing, $N / A$ means that it is overloaded, STABILIZING means that it is waiting for load to stabilize after load is exchanged, and BUSY means that it is currently in a load balancing session with another broker/cluster. A diagram showing the transition conditions between the local and global load balancing states is shown in Figures 6 and 7, respectively.

The local detection algorithm running on an edge broker is composed of two steps. Step 1 identifies whether the local broker is overloaded, and if so triggers load balancing. Step 2 is only invoked if Step 1 identifies no overload and no other observable edge brokers are overloaded. The latter condition gives overloaded brokers higher priority in establishing load balancing over nonoverloaded brokers that simply want to balance load. The purpose of Step 2 is to identify other edge brokers whose load difference with the local broker is greater by a threshold. Figure 8 summarizes the two phases of the local detection algorithm. 


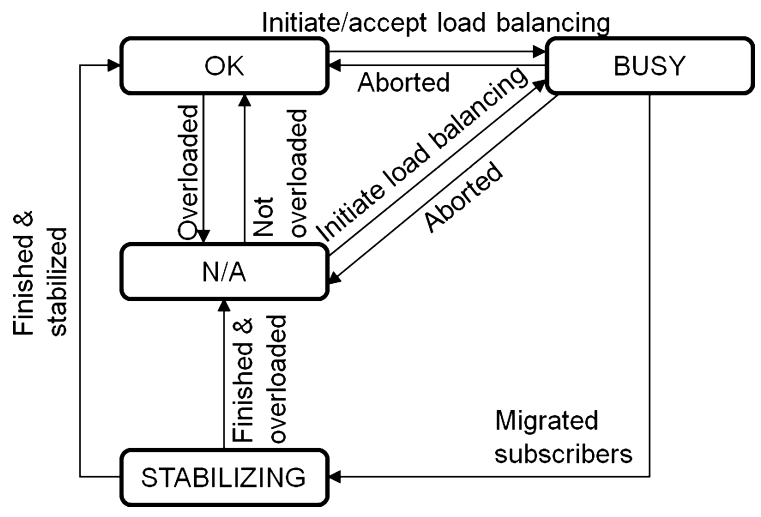

Fig. 6. State transition diagram for an edge broker.

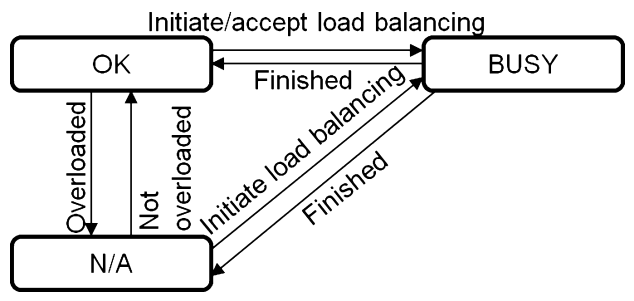

Fig. 7. State transition diagram for a cluster-head broker.

Specifically, Step 1 examines three utilization ratios, namely input, output, and CPU utilization ratio to determine if the broker itself is overloaded. The parameter lower overload threshold is introduced to prevent the broker from accepting further load by updating the broker's status to $N / A$ when one of its utilization ratios exceeds 0.9 . This parameter should be adjusted to reflect the maximum load under which the broker can operate reliably. If a utilization ratio exceeds the higher overload threshold at 0.95, then the broker is deemed close to overloading and load balancing is invoked immediately to start offloading subscriptions.

Both overload thresholds dictate the safe bounds under which a broker can consume the underlying physical resources. Very high overload threshold values will make more full use of the underlying resources, but the broker may enter the overloaded state before it has time to do offloading, which can have a significant impact on the delivery delay of messages. The magnitude of the difference between the two threshold parameters controls the efficiency of load balancing. The smaller the magnitude is, the more frequent the broker engages in load balancing sessions as a result of the following endless load balancing cycle: accept load from other brokers until reaching the lower overload threshold, trigger offloading due to reaching the higher overload threshold, accept load from other brokers until reaching the lower overload threshold and so on. However, a high magnitude in the difference will cause more load imbalance among the brokers. In summary, the higher overload threshold controls 


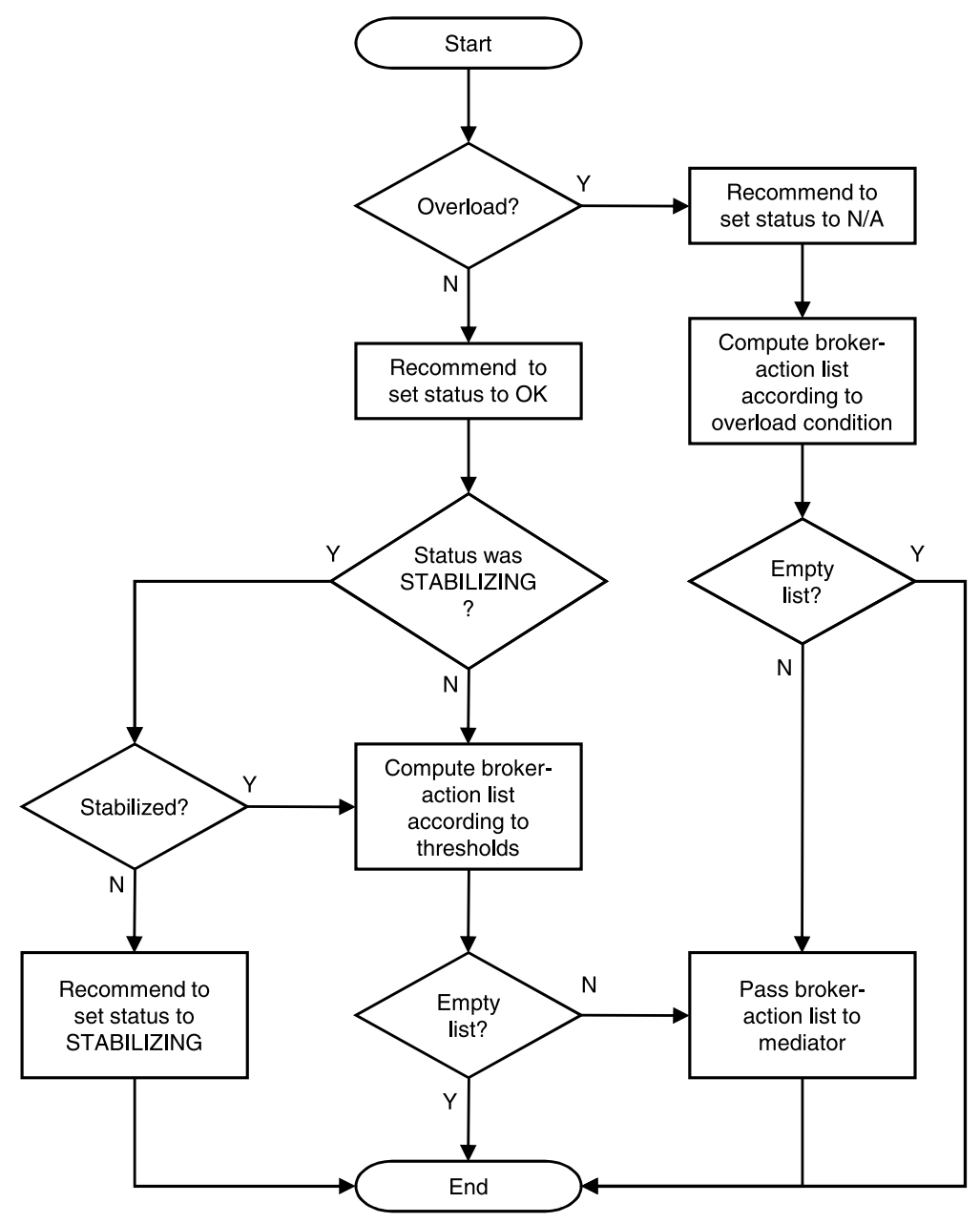

Fig. 8. Flowchart showing the local detection algorithm.

the tradeoff between the utility of the underlying physical resources and broker stability. The difference between the lower overload threshold and higher overload threshold controls the tradeoff between load balancing efficiency and load imbalance.

By the end of this step, a broker-action list of <load-accepting broker, offload action $>$ is generated. The list is sorted in descending order by the difference between the offloading broker's overloaded metric and load-accepting broker's corresponding metric. The offload action is "input" to denote triggering the input offload algorithm if the input utilization ratio is overloaded, "output" if the output utilization is overloaded, or "match" if the CPU utilization is overloaded. The reasoning behind the offload actions is that the input offload algorithm targets specifically at reducing the input utilization ratio, the output 
offload algorithm at reducing the output utilization ratio, and the match offload algorithm at reducing the matching/processing delay.

Step 2 checks to see if any one of the input utilization ratio, output utilization ratio, or matching delay differ from another edge broker by more than a threshold. The threshold for utilization ratios is called the local ratio trigger threshold, and for matching delay the local delay trigger threshold. The local ratio trigger threshold defines the minimum input or output utilization ratio difference before load balancing can be initiated between two brokers. Similarly, the local delay trigger threshold applies to the matching delay. All trigger thresholds have a value greater than 0 . A low trigger value will initiate load balancing on even the smallest load difference between two brokers. However, the trigger threshold should not be set lower than the load estimation error as that can lead to endless load balancing sessions trying to correct the imbalance arising from previous sessions. A high trigger threshold will yield more load imbalance among brokers, but since load balancing is rarely taking place, overhead is also lower as well. When the trigger threshold is equal to or higher than the higher overload threshold, load balancing only starts when a broker's input or output utilization is past the higher overload threshold. In general, the triggering thresholds control the tradeoff between the amount of overhead and the load imbalance tolerated by the algorithm. By default, both thresholds are set to 0.1 .

The difference for utilization ratio is just the magnitude of the difference, while for matching delay, the following formula is used:

$$
d \% \text { Diff }=\left|\frac{d_{1}-d_{2}}{N_{f}}\right|,
$$

where $d_{1}$ and $d_{2}$ are the two delay values used in the comparison. $N_{f}$ represents the normalization factor and is set to 0.1 by default so that delay differences much less than $0.1 \mathrm{~s}$ do not yield high percentage differences and trigger unwanted load balancing. At the end of this step, a broker-action list of <load-accepting broker, performance metric/offload action> is generated that is sorted in descending order of greatest performance metric difference. The purpose of sorting is to favor load balancing with load-accepting brokers that have the largest load difference. Not only does this potentially reduce the number of load balancing sessions to balance load across edge brokers, but also help to bring an overloaded broker out of its overloaded state in as few load balancing sessions as possible, thereby minimizing response time of the load balancing algorithm. The list is passed to the local mediator which establishes a load balancing session with the topmost available load-accepting broker in the list.

Immediately after a broker finishes a load balancing session, its load information may mislead the broker into making an incorrect load balancing decision. For example, brokers accepting load may not experience an increase in utilization immediately. This may cause the broker to accept more load balancing sessions, which may cause its resource consumption to overshoot. To prevent this from occurring, both the offloading and load-accepting brokers inherit a status of STABILIZING for a stabilize duration period before setting 
their status back to $O K$ (see Figure 6). When a broker has a STABILIZING status, it cannot accept load balancing requests nor invoke load balancing unless the broker is overloaded. If all performance metrics do not fluctuate by more than the stabilize percentage after a stabilize duration, then the broker sets its status back to $O K$.

The broker may wait for multiple stabilize duration periods if the stabilize percentage is not satisfied after each period. If the stabilize duration is set to a low value, then stability evaluation is done more frequently, which may help reduce waiting time and improve response time. However, a longer stabilize duration will capture a more accurate view of the broker's load, which leads to more reliable stabilization detection. In summary, this parameter controls the tradeoff between response time and load balancing effectiveness. By default, the stabilize duration is set to $30 \mathrm{~s}$, but it can be set to any other value as long as it allows sufficient time for the broker to sense its final load level.

As for the stabilize percentage, it should be set as low as possible to be certain that the broker's load has stabilized. However, in real deployments, the load of brokers is never constant even if the set of subscribers do not change and the publication rate of publishers stay constant. Some possible reasons for this include different publication-to-subscription matching patterns, shared server resources, network congestion, etc. Therefore, stabilize percentage should at the same time be set high enough so that noise does not prevent a broker from coming out of the stabilization phase. In summary, the stabilize percentage controls the tradeoff between effectiveness of load balancing and response time. By default, this parameter is set to 0.05 such that it is low enough to realize that load is no longer dropping drastically while ignoring noise.

Alternatively, in place of their utilization ratio counterparts, it is also possible for the load balancer to use input queuing delay and output queuing delay as performance metrics. However, queuing delays cannot show the utilization level of a resource but only indicate that the resource is overloaded when queueing delays rise rapidly. Moreover, queuing delay measurements do not accurately indicate the load of a broker at the instant the metric is measured because it is obtained after the message gets dequeued. Therefore, the measurement is lagging by the delay measured.

In the global detection algorithm, a cluster-head uses a subset of the status indicators in local load balancing (see Figure 7) to indicate its cluster's load balancing status. The only difference here is that a cluster is $N / A$ if one or more edge brokers are $N / A$. This allows the overloaded edge brokers to offload subscribers to brokers within the same cluster first to preserve locality. The global detection algorithm is almost the same as the local detection algorithm, except that the global detector uses different threshold values (namely, global ratio triggering threshold and global delay triggering threshold, both default to 0.15 ) and works with averaged values of each performance metric calculated from the edge brokers in the local cluster. As a rule of thumb, the global trigger thresholds should be set higher than the local trigger thresholds to prioritize load balancing among the edge brokers within the same cluster. The motivation behind this is to maintain the clients' connection within the same cluster 


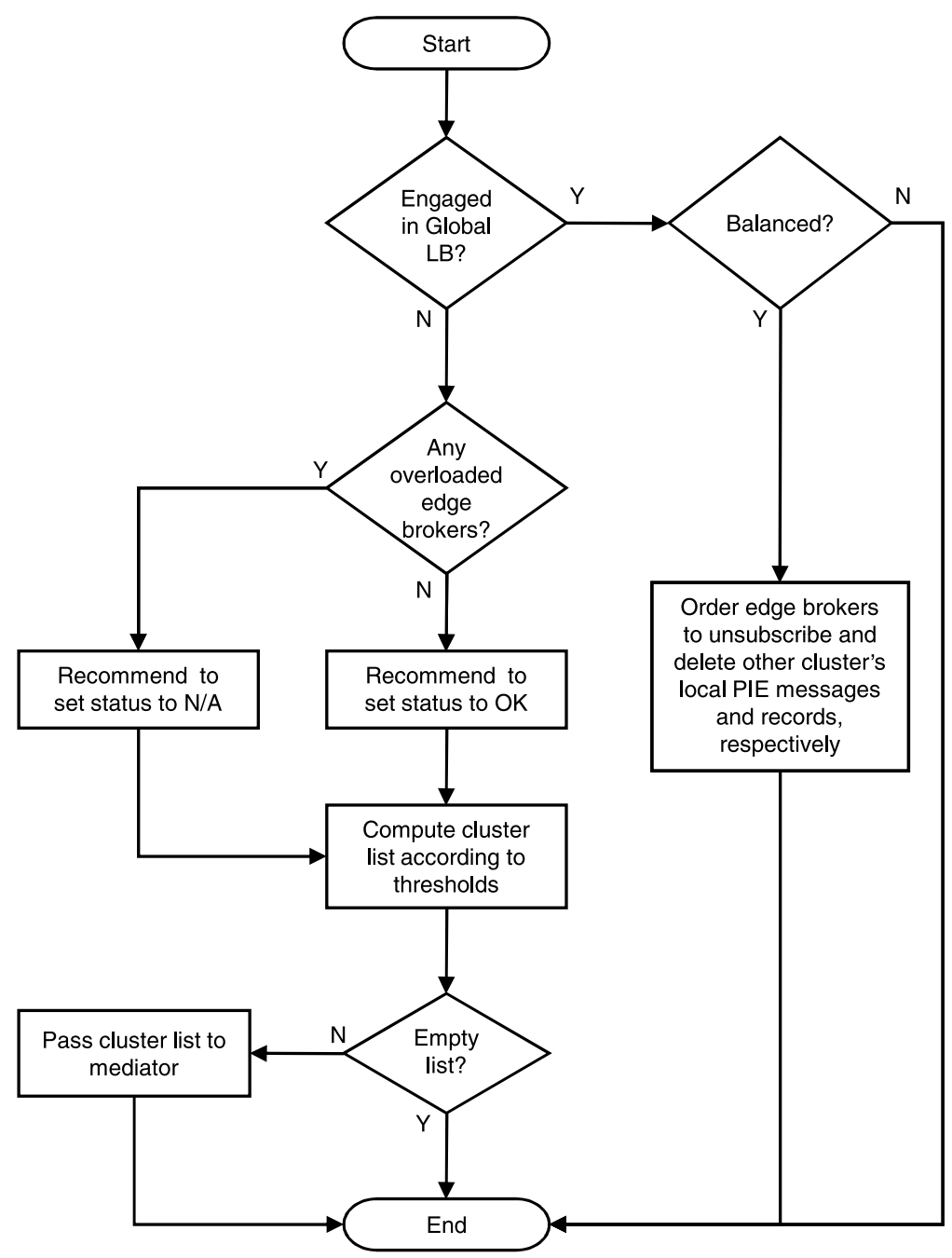

Fig. 9. Flowchart showing the global detection algorithm.

because the client is closest to that group of brokers in network vicinity (i.e., same network cluster or corporate network). Figure 9 summarizes the global detection algorithm.

Another way of invoking global load balancing is on receiving a number of global load balancing requests from one or more edge broker. An edge broker sends a global load balancing request to its cluster-head broker if it is in the $N / A$ state and cannot find another edge broker to load balancing with because none have a status of $O K$. The threshold for invoking global load balancing is defined by the parameter global load balancing request limit, whose default is set to 3. Setting this parameter to a low number will make the algorithm more responsive and minimize the amount of time an edge broker stays overloaded. 
However, because global load balancing is more easily triggered with a lower value, client locality is sacrificed. As well, message overhead increases due to increased frequency of global load balancing. In summary, the global load balancing request limit controls the tradeoff between responsiveness to overload, client locality, and overhead.

Both the local and global detector components run periodically to decide whether or not to trigger load balancing. Experiments in Cheung [2006] show that the periodic interval can be set very low to make the detector more responsive to overloads and load imbalance. However, since the detector makes decisions on the average load information gathered between each detection run, short detection intervals in the presence of temporary load spikes can lead to unnecessary load balancing, which may prevent convergence of the load balancing algorithm. Therefore, the detection interval should be set long enough so that the detector uses load information that better represents the broker's average load to make load balancing decisions. Instead of setting a fixed detection interval which may fix the load balancing order of edge brokers, we set the minimum and maximum interval values and let the brokers wait a random value between those bounds before subsequent detection runs. The respective minimum and maximum detection intervals for local load balancing are the local detection minimum interval and local detection maximum interval. The respective minimum and maximum detection intervals for global load balancing are the global detection minimum interval and global detection maximum interval. Generally, the minimum bound should not be set lower than half the maximum bound to ensure fairness among the brokers or clusters. As well, the global-level parameters can be set much higher than the local-level parameters to better preserve client locality and minimize overhead while trading off load imbalance among clusters. In summary, the detection interval parameters control the tradeoff between response time and load balancing effectiveness.

3.3.4 Adaptive PIE. From our experiments in running PIE at the default setting, the total message overhead is $0.2 \%$. However, if the PIE publishing frequency is increased (i.e., to decrease the response time of the load balancing algorithm), then the amount of message overhead will increase, which in turn will decrease the capacity of the system. To reduce the overhead of PIE messages at both local and global levels, we introduce Adaptive PIE that is an extension to the original PIE protocol outlined in Section 3.3.2. Adaptive PIE exploits the fine-grain filtering capability of content-based publish/subscribe to have brokers subscribe to only PIE messages that report a lighter load than the broker itself. This is achieved by dynamically adjusting the range of subscribed values for each of the three performance metrics. Instead of sending one subscription for local PIE messages, each broker sends three subscriptions, one for each of input utilization ratio, matching delay, and output utilization ratio. As well, each subscription has its own unique subscribed range. The following is an example of a local PIE subscription of a broker in cluster $C 1$ interested in input utilization ratio less than 0.5 :

[class, =, 'LOCAL_PIE'], [cluster, =, 'C1'], [input , <, 0.5] .

ACM Transactions on Computer Systems, Vol. 28, No. 4, Article 9, Pub. date: December 2010. 
Equation (5) shows how to compute the value to subscribe for any of the performance metrics.

$$
S_{\text {new }}^{x}=L_{\text {current }}^{x}-T_{L}^{x}+L_{\text {stddev }}^{x} \quad\left(0 \leq S_{\text {new }}^{x} \leq 1\right)
$$

Ideally, the upper bound value to subscribe on performance metric $x, S_{n e w}^{x}$, should always be less than or equal to the broker's current load value for metric $x\left(L_{\text {current }}^{x}\right)$ minus the metric's detection threshold $\left(T_{L}^{x}\right)$ as only those brokers can become potential load balancing candidates. However, since the load of the broker constantly changes over time, $S_{\text {new }}^{x}$ will have to be constantly updated through subscribe and unsubscribe operations, resulting in even more overhead than conventional PIE.

In order to avoid high overheads from frequent subscription updates due to broker load changes, we introduce some leeway into $S_{n e w}^{x}$, called $L_{\text {stddev }}^{x}$, which is the standard deviation of the broker's performance metric $x$ in the last $N$ published PIE messages. The use of a standard deviation function offers several benefits. First, if the broker's load is static, then $L_{\text {stddev }}$ approaches zero and the function $S_{\text {new }}^{x}$ is just $L_{\text {current }}^{x}-T_{L}^{x}$, which filters out unnecessary PIE messages and no subscription updates are required. Second, if a broker's load intermittently changes, then PIE subscription will only be updated intermittently at worse. Last, if the broker's load is very erratic, then $L_{\text {stddev }}^{x}$ dominates the equation, forcing $S_{\text {new }}^{x}$ to have an upper bound value of 1.0 over time, which also means no PIE subscription updates are necessary.

By default, $N$ has a value of $10 . N$ should be set high enough to capture past load fluctuation patterns at the broker, which will minimize re-subscribing of PIE messages. However, if the history is too long, the standard deviation may not be representative of the current load patterns, which may make the PIE subscription attract useless PIE messages from other edge brokers. Thus, the history should be set small enough to capture only recent load patterns. A shorter history also has the benefit of lower memory consumption and computational overhead. However, if the history is set too small, periodic load spikes in the past may not be captured by the standard deviation, which leads to unnecessary re-subscribing. To sum up, the history size controls the tradeoff between the number of PIE re-subscribing operations and the number of irrelevant PIE messages received.

A validity check for each performance metric's subscription is run at every PIE publication interval. If $S_{\text {new }}^{x}$ is greater than the current subscribed value $S_{\text {current }}^{x}$ by the detection threshold for this metric, then the subscription for this performance metric needs to be updated. On the other hand, if $S_{\text {new }}^{x}$ is less than $S_{\text {current }}^{x}$, then the subscription for this performance metric is only updated if $S_{\text {current }}^{x}$ is greater than at least one of the other edge broker's corresponding performance metric and $S_{\text {new }}^{x}$ is less than that same figure. In other words, do not update the subscription unless the new subscription can filter out PIE messages from at least one other broker. On bootstrap, the broker uses a value of 0 for $S_{n e w}^{x}$. Our experiments in Section 6.3.4 show that Adaptive PIE indeed reduces the amount of PIE-related message overhead by up to $65 \%$. 


\subsection{Mediation Protocols}

All load balancing activities are coordinated by exchanging messages using the underlying publish/subscribe infrastructure for simplicity and efficiency. Specifically, request-reply and one-way protocols are implemented in publish/subscribe to coordinate broker and subscriber activities.

3.4.1 Mediating Load Balancing Sessions. A local load balancing session consists of a pair of brokers: an offloading broker and a load-accepting broker. Once a broker is engaged in a local load balancing session, it cannot participate in another local load balancing session until the current one is finished. This is to allow the offloading broker to accurately estimate the load impact of offloading subscription to the load-accepting broker. An alternative design choice may involve one offload broker with multiple load-accepting brokers. However, such a design can introduce deadlocks if two or more offloading brokers attempt to "reserve" the same set of load-accepting brokers. Even with deadlock prevention, offloading brokers may be required to serialize their local load balancing sessions, which slows down the response time of the algorithm. With our pairwise load balancing approach, pairs of brokers can load balance concurrently without deadlocking, making the algorithm efficient and simple, which is inline with the goals that commercial systems strive to achieve.

A local load balancing session is composed as follows. Once the local detection algorithm composes the broker-action list of candidate brokers for load balancing, the local mediator sends a load balancing request sequentially to brokers in the sorted list. When a load-accepting broker gets this request, its local mediator replies back with its current status. If the status is $O K$, the request is accepted and both brokers update their status to BUSY. In the $O K$ reply, the load-accepting broker appends its load information in the message so that the requesting broker can use this information for offload algorithms to compute which subscribers are suitable for offload. This load information includes the load-accepting broker's CSS, input publication rate, matching delay equation, number of subscriptions, and total output bandwidth. For all other states, the load-accepting broker rejects the load balancing request.

A global load balancing session consists of a pair of clusters where edge brokers from each cluster load balance with each other. A pairwise design follows the same efficient and simple design theme as the local load balancing approach. The global mediator running at the cluster-head broker uses the same protocol as the local mediator to set up global load balancing. The difference here is that after a successful handshake, both cluster-heads have to tell all edge brokers in their own cluster to subscribe to the other cluster's local PIE messages. This allows edge brokers from one cluster to load balance with edge brokers in the other cluster.

Global load balancing ends when all edge brokers are balanced with each other as indicated by the balanced set field in local PIE messages. On terminating a global load balancing session, edge brokers unsubscribe from the local PIE publications of the other cluster. This action has the effect of blocking edge brokers in one cluster from seeing edge brokers in another cluster through PIE 
messages, which in turn stops local load balancing sessions between two clusters from initiating.

3.4.2 Mediating Subscriber Migration. Once the offloading algorithm is done with its computation, it returns back to the mediator a list of subscribers to offload. The mediator has to migrate the indicated subscribers to the new broker in the most efficient and timely manner with minimal delivery loss. First, the mediator sends a control publication message to each subscriber in the offload list telling them to issue their subscription to the new load-accepting broker. Subscribers issue a subscription to the load-accepting broker containing the ID of the load balancing session and the total number of migrating subscribers. These two pieces of information allow the load-accepting broker to know when it has received all migrating subscribers in the current load balancing session. For efficiency and best-effort guarantee of minimal delivery loss, the receiving broker waits for $N \times$ migration timeout seconds for all migrating subscribers to connect, where $N$ is the total number of migrating subscribers, and migration timeout is set to a default value of $5 \mathrm{~s}$. A high timeout value will ensure that subscribers that are slow to connect to the load-accepting broker will not lose any publication messages throughout the migration process. However, a high timeout value will make the load-accepting broker wait unnecessarily long for clients that disconnected from the system or crashed during migration.

While subscribers are connected to the two brokers, they need to detect and drop duplicate publications (by using a short message history) because they are subscribing to the same subscription at two different endpoints. When all subscribers have connected to the load-accepting broker or when the timeout occurs, the receiving broker sends a DONE control publication message back to the offloading broker to terminate the load balancing session. This message ensures that the publication paths for all migrated subscribers have been set up to flow to the load-accepting broker. When the offloading broker receives the DONE message, it tells the migrating subscribers to wait for all the messages currently in the offloading broker's input queue to be matched and delivered from the output queues before sending an unsubscribe message. This waiting period corresponds to the sum of the offloading broker's input queuing delay, matching delay, and output queuing delay. Once the migrating subscribers unsubscribe from the offloading broker, the migration process is complete. Note that all control and duplicate messages are handled transparently by a thin software layer on the client side that hides the intricate details of load balancing from the end-user application.

\section{LOAD ESTIMATION ALGORITHMS}

Load estimation is used by the offload algorithms to estimate a subscription's load contribution in the form of additional input publication rate, matching delay, and output publication rate on the load-accepting broker as well as the load reduced at the offloading broker.

ACM Transactions on Computer Systems, Vol. 28, No. 4, Article 9, Pub. date: December 2010. 


\subsection{Estimating Load Requirements of Subscriptions}

Publish/subscribe Real-time Event-to-Subscription Spectrum ${ }^{4}$ (PRESS) is a space and time-efficient technique for estimating the bandwidth requirements and common set of publication messages attracted by two or more subscriptions based on current events. It uses bit vectors to record the matching pattern of subscriptions, hence the term event-to-subscription. It does not require the publish/subscribe system to use advertisements, nor does it assume that publications are in any sort of distribution. The operation of PRESS is best explained as part of the local load balancing algorithm after the mediation step as described in Section 3.4.1 where two brokers have agreed to load balance with each other.

First, the offloading broker locally subscribes to the CSS of the loadaccepting broker (as supplied in the $O K$ reply message from the replying broker). Locally subscribe means that subscriptions are sent to the matching engine, but never get forwarded to neighboring brokers because their message TTL is set to 0 . The purpose of this operation is for the offloading broker to identify which publications it currently sinks are also received by the load-accepting broker. Next, all client subscriptions in the matching engine are allocated a bit vector of length $N_{p}$ initialized to 0 , where $N_{p}$ represents the number of samples. Sampling starts immediately after getting the load-accepting broker's $O K$ reply message and ends after $N_{p}$ publications have been received or a timeout $T$ is met, whichever comes first. According to our micro experiments in Cheung [2006], a low sample limit will make PRESS more responsive, but the load estimation accuracy will suffer below 50 samples. As well, a high sample limit beyond 100 not only makes PRESS slow, but also hurts estimation accuracy. By default, $N_{p}$ is set to $50 . T$, on the other hand, is used to limit the time spent on profiling load under the condition where the broker's incoming publication rate is very low. If one favors estimation accuracy without regard for response time, then $T$ should be set to an extremely high value. $T$ has a default value of $30 \mathrm{~s}$.

The algorithm starts at the rightmost position of the bit vector for all subscriptions. A ' 1 ' is set if the subscription matched the incoming publication, ' 0 ' otherwise, before moving onto the next bit on the left. During the sampling period, the total incoming publication rate is measured. Table $\mathrm{I}(\mathrm{a})$ and $\mathrm{I}(\mathrm{b})$ show an example of the bit vectors measured at the offloading broker with $N=6$ for subscriptions at the offloading broker and load-accepting broker, respectively, given the following publication arrival order:

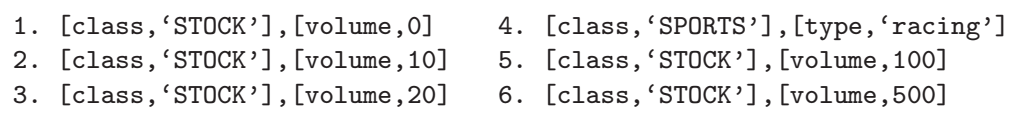

Equation (6) shows the formula to calculate the publication rate matching a particular subscription represented by $s_{P R}$, where $i_{r}$ represents the total input publication rate of the offloading broker, $n_{B S}$ represents the number of bits set

${ }^{4}$ Real-time refers to sampling using live incoming publications to the broker. ACM Transactions on Computer Systems, Vol. 28, No. 4, Article 9, Pub. date: December 2010. 
Table I. Bit Vector Example

\begin{tabular}{|c|c|}
\hline \multicolumn{2}{|c|}{ (a) Candidate subscriptions to offload } \\
\hline Candidate Subscriptions & Bit Vector \\
\hline [class, , , 'STOCK'] & 110111 \\
\hline [class, =, 'STOCK'], [volume,>,15] & 110100 \\
\hline [class, , , 'STOCK'], [volume, , , 150] & 100000 \\
\hline [class, , , 'SPORTS'] & 001000 \\
\hline \multicolumn{2}{|c|}{ (b) Load-accepting broker's CSS } \\
\hline Load-Accepting Broker's CSS & Bit Vector \\
\hline [class, , , 'STOCK'], [volume, ,>,50] & 110000 \\
\hline [class, =, 'STOCK'], [volume,$<, 5]$ & 000001 \\
\hline [class , =, 'MOVIES'] & 000000 \\
\hline CSS bit vector & 110001 \\
\hline
\end{tabular}

in the subscription's bit vector, and $N$ represents the number of samples taken in PRESS.

$$
s_{P R}=i_{r}\left(\frac{n_{B S}}{N}\right)
$$

For example, if the total input publication rate $i_{r}$ at the offloading broker is assumed to be $3 \mathrm{msg} / \mathrm{s}$, and if the subscription [class, =, 'STOCK'] is assumed to have 5 out of the 6 bits set, then the publication rate for [class, =, 'STOCK'] comes out to $2.5 \mathrm{msg} / \mathrm{s}$. Moreover, the additional incoming publication rate introduced at the load-accepting broker for each candidate subscription can be calculated by using Eq. (6) with $n_{B S}$ obtained from the following function:

$$
n_{B S}=\left|s_{B V} \wedge \overline{l_{c s s B V}}\right|
$$

where $s_{B V}$ is the candidate subscription's bit vector and $l_{c s s} B$ is the aggregated load-accepting broker's CSS bit vector. The intuition behind Eq. (7) is that we are interested in the set publications that are received by the offloading broker and, at the same time, not received by the load-accepting broker. For example, to calculate the additional incoming publication rate imposed by the subscription [class, =, 'STOCK'] on the load-accepting broker, we first compute the value of $n_{B S}$

$$
n_{B S}=|110111 \wedge \overline{110001}|=|000110|=2 .
$$

With $n_{B S}$ of 2 , and reusing $3 \mathrm{msg} / \mathrm{s}$ for $i_{r}$, the additional incoming publication rate on the load-accepting broker for this subscription is $1 \mathrm{msg} / \mathrm{s}$. In some cases, offloading a subscription may alter the CSS of the load-accepting broker. With PRESS, it is not necessary to resample all subscriptions again because the aggregated CSS bit vector can be updated by merging it with the offloaded subscription's bit vector using the $O R$ bit operator. For example, if [class, =, 'STOCK'] was chosen for offloading, then the load-accepting broker's CSS is updated to 110111.

Given $S$ candidate subscribers, $C$ subscriptions in the load-accepting broker's CSS, and $N$ publications to sample, the memory overhead of PRESS is $\mathrm{O}((S+$ $C) N$ ) bits and the matching overhead is $\mathrm{O}(S+C)$. Under typical deployments 
where there are 10,000 subscribers with $N$ set to at most 100 , PRESS only uses $1 \mathrm{Mb}$ of memory. Given that the load-accepting broker's CSS is usually small (it is just one in the case of $[\mathrm{class},=, *]$ ), an increase in the matching delay is negligible.

\subsection{Modeling and Estimating Performance Metrics}

In this section, we describe an approach to model the matching delay and how to estimate gains or reductions in matching delay, input utilization ratio, and output utilization ratio at the offloading and load-accepting brokers.

Depending on the matching algorithm and workload that is used, the matching delay may grow linearly or exponentially with the number of subscriptions stored in the matching engine. To decouple our load balancing algorithm with the implementation of the matching algorithm, we chose to use a linear equation to model the matching delay, $d$ :

$$
d=m n+b
$$

where $m$ is the slope of the matching delay function, $n$ is the number of subscriptions, and $b$ is the y-intercept. The advantages of using a linear equation is in its simplicity and its ability to accurately capture the slope of any matching delay function given a specific number of subscriptions in the matching engine. The disadvantage is that it is unable to accurately model a nonlinear matching delay function over a wide range of $n$. To remedy this shortcoming, multiple linear functions across different ranges of $n$ will be used to model an arbitrary matching delay function. This is done by calculating values for $m$ and $b$ using Eq. (9) over different number of subscriptions in the past. The number of subscriptions is rounded to the nearest ten to capture delays over wider range in subscription count. In other words, matching delays for subscription counts from 5-14 are assigned to bucket "10", 15-24 are assigned to bucket " 20 ", etc. To avoid storing too many readings, the maximum number of buckets is limited to 10, where each bucket stores a running average of the observed matching delay for a specific subscription count. A bucket is only created when the first matching delay value is observed and no buckets exist for that specific subscription count. Since the number of subscriptions tend to increase over time, buckets with the least subscription count are booted if the total number of buckets exceed 10. A maximum of 2 consecutive delay values that surpass $150 \%$ or undercut $50 \%$ of the last observed 5 or more values are ignored to filter out spikes due to uncontrollable external disturbances such as Java's garbage collector or external processes on PlanetLab. With two or more buckets filled, the variable $m$ is calculated by averaging the slope values from taking the last updated bucket with all other buckets. With $m$, the variable $b$ is calculated by substituting $d$ and $n$ from the last updated bucket. The reason for choosing the last updated bucket is so that the final equation contains that point, which is also the best indication of the broker's current performance. With both variables $m$ and $b$ known, the matching delay can easily be estimated by substituting a different number for $n$. 
Input utilization ratio $\left(I_{r}\right)$ is estimated by substituting estimated values into the variables of the input utilization ratio equation (Eq. (1)):

$$
I_{r}=\frac{i_{r}^{\prime}}{m_{r}^{\prime}}
$$

where $i_{r}^{\prime}$ is the new rate of incoming publications estimated using PRESS, and $m_{r}^{\prime}$ is the new maximum message match rate calculated by taking the inverse of the estimated matching delay. Since CPU utilization ratio is the equivalent of this load index when the value is less than 1 , this formula is also used for predicting CPU load.

Output utilization ratio $\left(O_{r}\right)$ is estimated by substituting estimated values into the variables of the output utilization ratio equation (Eq. (2)):

$$
O_{r}=\frac{o_{u}^{\prime}}{o_{t}}
$$

Since the total output bandwidth is fixed, the only missing variable here is the estimated output bandwidth usage $\left(o_{u}^{\prime}\right)$, which is given by:

$$
o_{u}^{\prime}=o_{u}+\Delta o_{u}
$$

where $\Delta o_{u}$ is the change in output traffic imposed by the offloaded subscribers estimated using PRESS.

\section{OFFLOAD ALGORITHMS}

After profiling all subscriptions using PRESS, the offloading broker will feed the profiled data along with the load-accepting broker's load information to the offload algorithm to compute the set of subscribers to offload. The offload algorithm to choose depends on what performance metric to balance, which is decided initially by the detector in the broker-action list as mentioned in Section 3.3.3. Table II summarizes the key properties of all offload algorithms.

For baseline comparison against the three offload algorithms, we introduce a naive offload algorithm called the Random Algorithm to load balance the three performance metrics. The purpose of introducing this algorithm is to show the effectiveness of load balancing a content-based publish/subscribe system with neither load estimation nor subscription space awareness. Section 5.4 will describe more details about this algorithm.

\subsection{Input Offload Algorithm}

This algorithm is invoked by the offloading broker when the input utilization ratio needs load balancing. The aim here is to reduce the offloading broker's input utilization ratio and increase the same metric on the load-accepting broker with minimal effect on the other performance metrics. There are two strategies to reduce the input utilization ratio: increase the rate at which messages are matched, or reduce the rate of incoming publication messages. Increasing the rate of matching is achieved by reducing the number of subscriptions in the matching engine. However, this action conflicts with the match offload algorithm that is trying to balance the matching delay and therefore is not applied

ACM Transactions on Computer Systems, Vol. 28, No. 4, Article 9, Pub. date: December 2010. 
Table II. Properties of All Offload Algorithms

\begin{tabular}{|c|c|c|c|}
\hline $\begin{array}{l}\text { Offload } \\
\text { Algorithm }\end{array}$ & $\begin{array}{c}\text { Performance Metric } \\
\text { Being Balanced }\end{array}$ & Methodology & Side Effects \\
\hline Input & Input utilization ratio & $\begin{array}{l}\text { Offload subscriptions } \\
\text { in the CSS }\end{array}$ & $\begin{array}{l}\text { Output utilization ratio } \\
\text { is also decreased at } \\
\text { offloading broker and } \\
\text { increased at load- } \\
\text { accepting broker }\end{array}$ \\
\hline Match & $\begin{array}{l}\text { Matching delay } \\
\text { Overloaded CPU } \\
\text { utilization ratio } \\
\text { Overloaded memory } \\
\text { utilization ratio }\end{array}$ & $\begin{array}{l}\text { Offload subscriptions } \\
\text { with least traffic }\end{array}$ & None \\
\hline \multirow[b]{2}{*}{ Output } & \multirow[b]{2}{*}{ Output utilization ratio } & $\begin{array}{l}\text { Offload subscriptions } \\
\text { with highest traffic } \\
\text { in Phase-I }\end{array}$ & None \\
\hline & & $\begin{array}{l}\text { Offload subscriptions } \\
\text { with highest traffic } \\
\text { and minimal side- } \\
\text { effects in Phase-II }\end{array}$ & $\begin{array}{l}\text { Increases input } \\
\text { utilization ratio of } \\
\text { load-accepting broker }\end{array}$ \\
\hline Random & All & $\begin{array}{l}\text { Offload randomly } \\
\text { chosen subscriptions }\end{array}$ & $\begin{array}{l}\text { Unpredictable, oscillations } \\
\text { may occur }\end{array}$ \\
\hline
\end{tabular}

here. Hence, the incoming publication rate can only be reduced by offloading subscriptions in the CSS because their subscription space is a superset of all subscriptions not in the CSS. With the poset [Carzaniga et al. 2001], CSS lookup will take $\mathrm{O}(1)$ time. Once the subscriptions in the CSS are identified, a report card is calculated for each of them. A report card consists of the following fields:

(1) Number of subscribers of this subscription to offload.

(2) Resulting load percentage difference between the two brokers by offloading this subscription, where a negative value indicates that the offloading broker will become less loaded than the load-accepting broker. This value is calculated using the estimated input utilization ratios of the two brokers in the input offload algorithm, matching delays in the match offload algorithm, and output utilization ratios in the output offload algorithm.

(3) Boolean value indicating if this subscription is covered by the loadaccepting broker's CSS.

(4) Publication rate reduced at the offloading broker estimated using PRESS.

(5) Output bandwidth required per subscriber estimated using PRESS.

The number of subscribers to offload per unique subscription is restrained by two conditions. First, the offload should not overload any of the load-accepting broker's resources. Second, the performance metric of interest of the two brokers should be balanced within the balanced threshold, which is 0.005 by default; or bring the offloading broker's metric below the load-accepting broker's. The performance metric of interest for the input, match, and output offload algorithms are the input utilization ratio, matching delay, and output utilization ratio, respectively. 
Specifically, the balance threshold parameter controls how closely an offload algorithm will attempt to balance the performance metric of two brokers in a local load balancing session. The value of this parameter can be any real number equal to or greater than 0 . By setting the parameter to zero, the offload algorithm will offload subscriptions until the difference between the two brokers' performance metric is zero or until any further offload from the offloading broker will make the load-accepting broker's performance metric become higher than the offloading broker's. If the parameter is a non-zero value, then the offload algorithm will offload subscriptions until the difference between the two brokers' performance metric is within the defined threshold. It is possible for the load-accepting broker to end up having a higher load than the offloading broker in this case. Therefore, setting the balance threshold too high may cause the load balancing algorithm to diverge. In summary, this parameter controls the tradeoff between load balancing effectiveness and load balancing stability.

After calculating the report cards to determine the number of subscribers to offload for each subscription in the CSS, the subscription that results in the two brokers' input utilization ratio difference closest to zero is chosen for offloading. This selection scheme ensures that subscriptions with the highest input publication rate are chosen first, which helps to reduce the number of subscriptions offloaded, and in so doing, reduces the impact on the load-accepting broker's matching delay. If any additional offload will result in a higher load difference than before, then the selection process terminates. This guarantees that all load balancing actions will always converge to a state where the brokers have smaller load differences.

Subscriptions chosen to be offloaded are removed from the poset to prevent future consideration for offloading. Load information of both brokers (the one obtained in the mediation process) is updated with estimated values according to the offloaded subscription's report card. Updated load information of both brokers are used on the next iteration of the subscription selection algorithm. The selection process ends when no more subscriptions are available for offloading, the offloading broker's input utilization ratio is below that of the load-accepting broker, or the absolute difference between the two brokers' input utilization ratios fall within the balance threshold.

The computational complexity of the input offload algorithm is $\mathrm{O}\left(n^{2} \log n\right)$ where $n$ is the number of subscriptions at the offloading broker. A summary of the input offload algorithm is given in Algorithm 1 in the form of pseudocode.

\subsection{Match Offload Algorithm}

Although the input utilization ratio varies directly with the matching delay, balancing the input utilization ratio does not balance the matching delay. The objective of this offload algorithm is to balance the matching delays without affecting the input and output utilization ratios of the two brokers. Intuitively, subscriptions with the lowest publication traffic are most suited to this criterion. Furthermore, subscriptions that introduce the smallest amount of incoming traffic into the load-accepting broker are most favorable. In this algorithm, report cards are computed for all subscriptions in the offloading broker and

ACM Transactions on Computer Systems, Vol. 28, No. 4, Article 9, Pub. date: December 2010. 


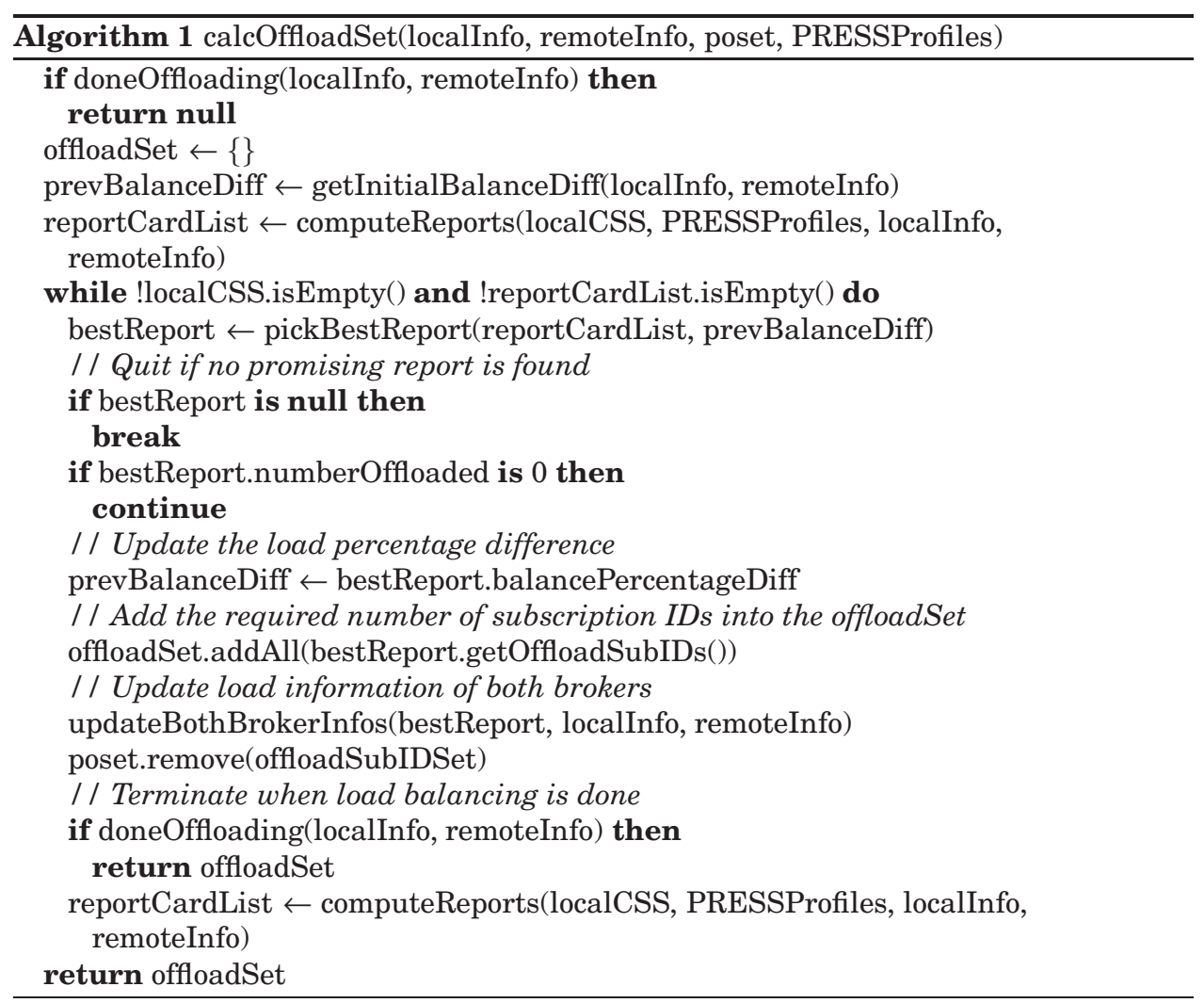

they are sorted by ascending output bandwidth. The algorithm to compute number of subscribers to offload for each unique subscription is almost identical to the input offload algorithm. The only difference is that input utilization ratios are replaced by matching delays.

If the match offload algorithm is invoked because the broker is overloaded and wants to reduce its CPU utilization ratio, input utilization ratio, or memory utilization ratio, then subscriptions should continue to be offloaded until the CPU utilization ratio, input utilization ratio, and memory utilization ratio drops below the lower overload threshold. After a subscription is chosen to be offloaded, load information about both brokers are updated. The same criterion used in the input offload algorithm applies here for terminating the match offload process. The computational complexity of the match offload algorithm is $\mathrm{O}\left(n^{2} \log n\right)$ where $n$ is the number of subscriptions at the offloading broker. This offload algorithm follows the same framework as the input offload algorithm summarized in Algorithm 1.

\subsection{Output Offload Algorithm}

This algorithm attempts to balance the output utilization ratios of two brokers by manipulating the amount of output bandwidth used at each broker. 


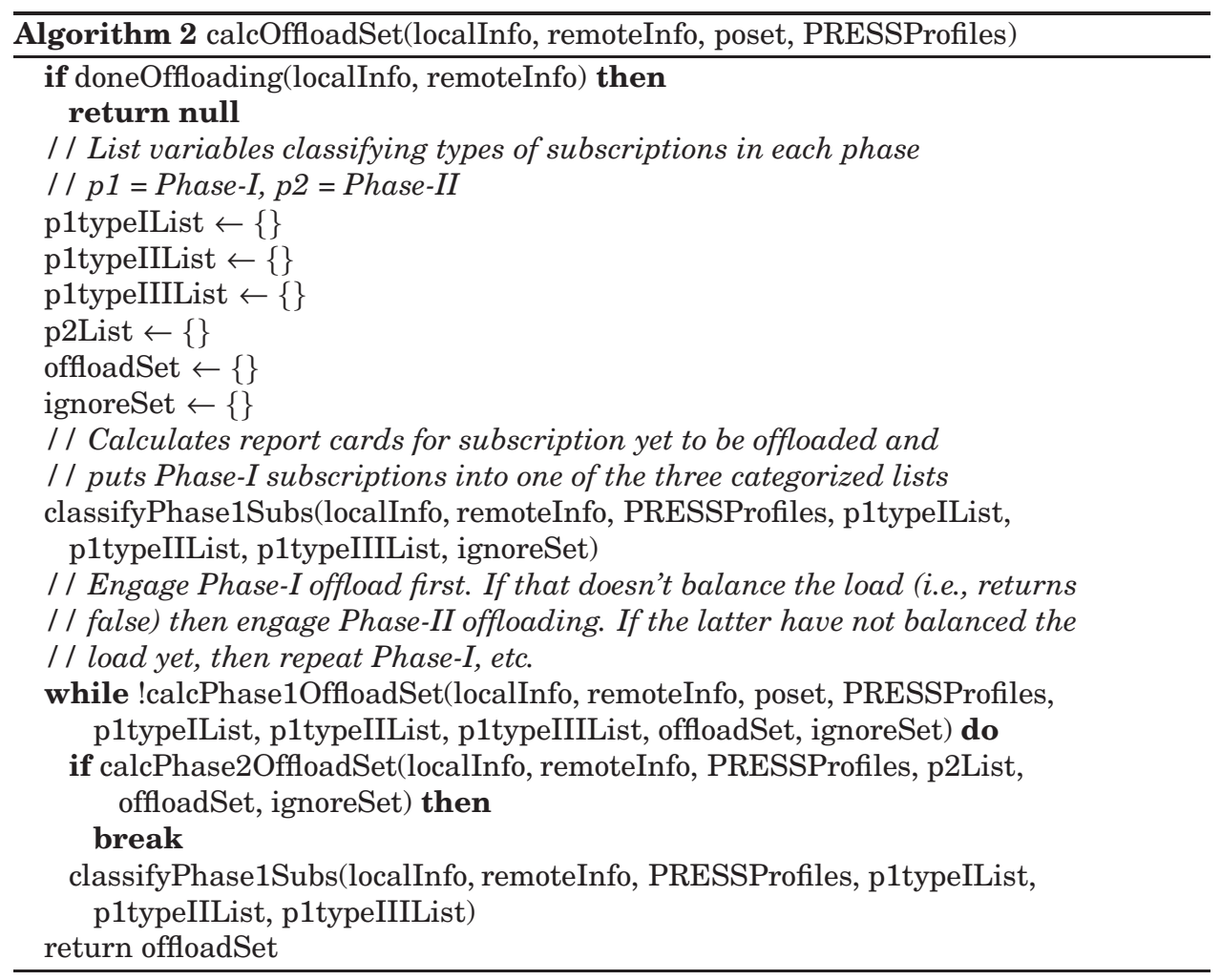

Prioritizing subscriptions for the offload process is divided into two phases. In Phase-I, subscriptions that are covered by or equal to the load-accepting broker's CSS are considered. These subscriptions are further classified into three types by using the fields computed for every subscription's report card. Offloading Type-I subscriptions will reduce the input publication rate of the offloading broker. These should be offloaded first because they reduce the overall input load of the system. Type-II subscriptions are similar to Type-I, except that they do not reduce the input publication rate because all subscribers for a subscription cannot be offloaded to produce a more balanced state. Type-III subscriptions are considered last in Phase-I because they do not reduce the input publication rate of the offloading broker even if all subscribers for that particular subscription are offloaded. The algorithm for calculating the number of subscribers to offload for each unique subscription is similar to the input offload algorithm shown previously, except that input utilization ratios are now replaced by output utilization ratios.

After a subscription is chosen to be offloaded, load information about both brokers is updated. If both brokers are balanced, then the algorithm stops and forwards the subscriber migration list to the mediator. Otherwise, Phase-II is invoked to further balance the output utilization ratio with some side-effects. All subscriptions considered in Phase-II are not contained in the CSS of the load-accepting broker. Therefore, these subscriptions may have the side-effect 


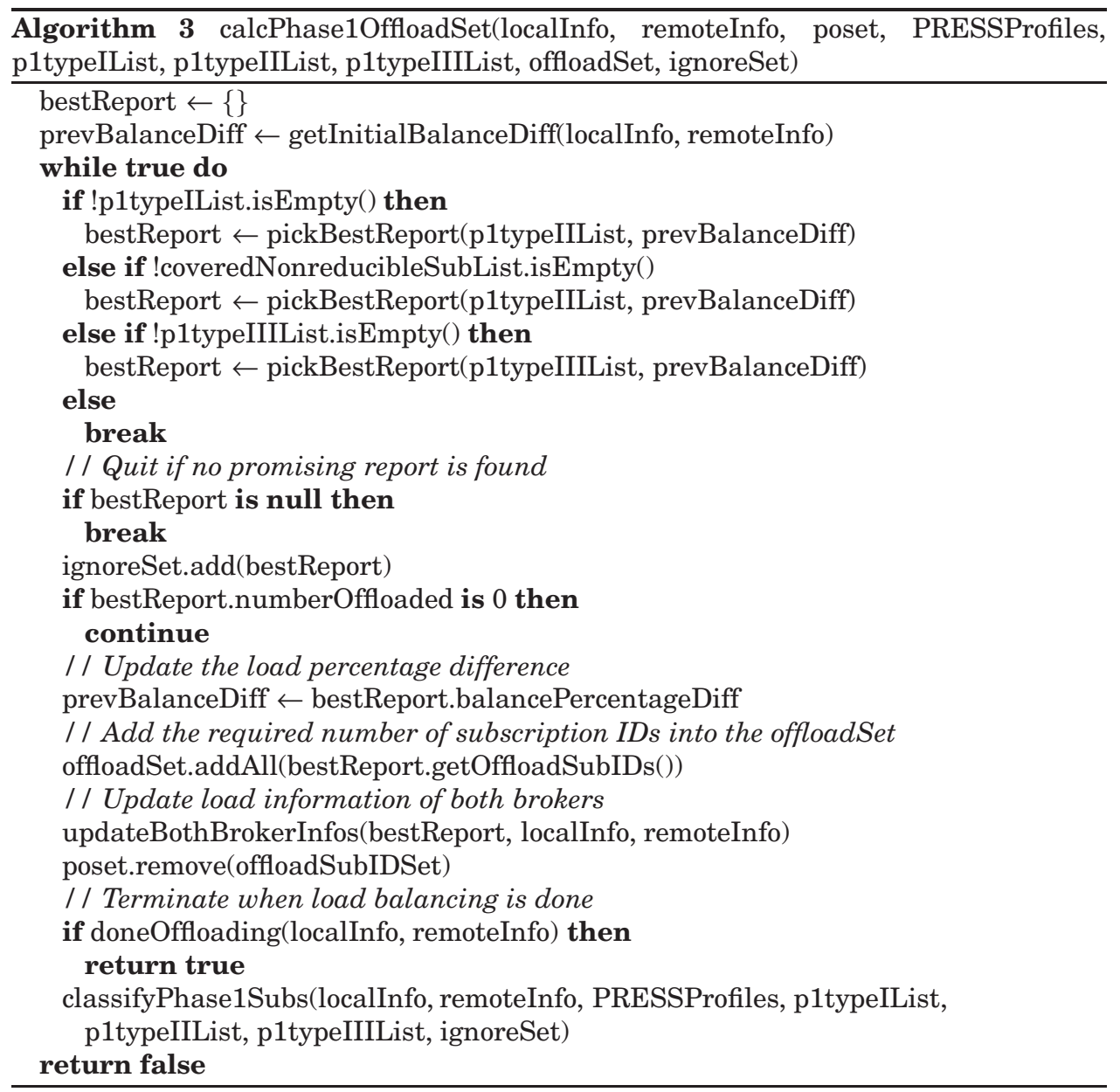

of significantly increasing the incoming publication rate of the load-accepting broker. What may happen is that there will be an oscillation between the input offload algorithm trying to balance the input utilization ratio disrupted by Phase-II of the output offload algorithm, and Phase-II of the output offload algorithm trying to balance the output utilization ratio disrupted by the input offload algorithm. To prevent this unstable situation from happening, Phase-II terminates when the input utilization ratios of both brokers are balanced, even if the output utilization ratios are not. An exception applies if the offloading broker is output overloaded, in which case the offloading broker will stop offloading once its output utilization ratio is below the lower overload threshold. With this exception, no oscillation occurs because the offloading broker cannot take back any subscriptions since it has a status of $N / A$ at the lower overload threshold.

The sorting and selection scheme in Phase-II is exactly the same as in the input offload algorithm with the use of load differences. If the subscription offloaded in Phase-II covers other local subscriptions, then Phase-I is invoked 


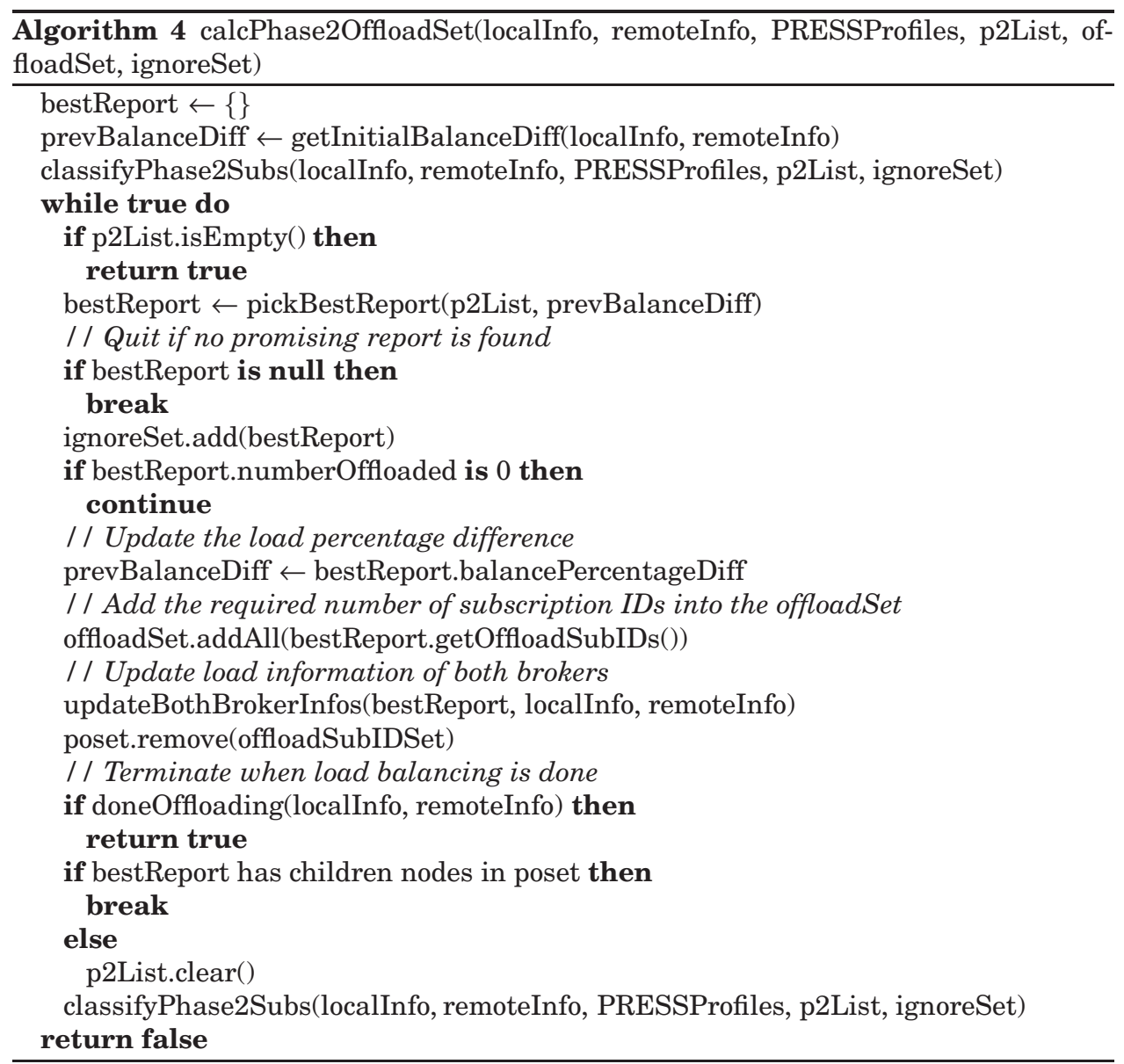

to offload those covered subscriptions because they are now covered by the loadaccepting broker's CSS. Otherwise, if the subscription offloaded in Phase-II does not cover any other subscriptions, then Phase-II continues to run. The computational complexity of the output offload algorithm is $\mathrm{O}\left(n^{2} \log n\right)$ where $n$ is the number of subscriptions at the offloading broker. The output offload algorithm is summarized by pseudocode in Algorithm 2 with details of Phase-I and Phase-II summarized in Algorithms 3 and 4, respectively.

\subsection{Random Algorithm}

The Random Algorithm is a naive approach to load balance any of the three performance metrics. Since the purpose of this algorithm is to represent a generic load balancer, it has no load estimation and no awareness of subscription space. Instead, this algorithm uses simple calculations to determine the number of subscriptions to offload, $c$, then randomly picks $c$ subscriptions to offload. This effectively gives the algorithm a runtime complexity of $\mathrm{O}(c)$. It is possible to make the subscription count random as well, but this will make the 
algorithm too naive and inefficient to draw a meaningful comparison with the three offload algorithms.

To compute the offload subscription count, the offloading broker first computes the target value to reach for the chosen performance metric $x$. The target value, $L_{x}^{a v g}$, is simply the average of the offloading broker's value of the performance metric, $L_{x}^{o f f}$, and the load-accepting broker's value of the same performance metric, $L_{x}^{a c c}$. Then, Eq. (13) below is used to calculate $c_{1}$, the number of subscriptions to offload, with $n_{\text {off }}$ representing the number of subscriptions at the offloading broker itself.

$$
c_{1}=n_{o f f}-L_{x}^{a v g}\left(\frac{n_{o f f}}{L_{x}^{o f f}}\right)
$$

However, using $n_{\text {acc }}$ (the number of subscriptions residing at the loadaccepting broker) yields a different result:

$$
c_{2}=L_{x}^{a v g}\left(\frac{n_{a c c}}{L_{x}^{a c c}}\right)-n_{a c c} .
$$

A simple solution is to simply take the average of $c_{1}$ and $c_{2}$ to arrive at $c$. Or set $c$ to be $c_{1}$ if $c_{2}$ is greater than the maximum number of subscriptions at the offloading broker.

Because the random algorithm is not aware of the potential oscillating effect of offloading certain subscriptions (such as those in Phase-II of the output offload algorithm as described above) and because it has no load estimation mechanism, the effect of each offload is unpredictable and may lead to load oscillations. Oscillations in turn cause the algorithm to diverge which cause instability in the system as witnessed in our experiments on heterogeneous platforms.

\section{EVALUATION}

For experimentation, we implemented all approaches presented in this article on the open source PADRES [Cheung and Jacobsen 2006; Li and Jacobsen 2005; Li et al. 2007; MSRG 2008] distributed content-based publish/subscribe system. We ran experiments on homogeneous and heterogeneous platforms including PlanetLab and a cluster testbed to compare how the load balancing algorithm performs in a real setting versus simulation setting. Our results will examine the effectiveness and efficiency of load balancing at both the local and global levels.

\subsection{Implementation}

The proposed load balancing solution, including extensions such as Adaptive PIE, the Random Algorithm, and a simulator, are implemented with 36,000 lines of Java code on top of PADRES [Cheung and Jacobsen 2006; Li and Jacobsen 2005; Li et al. 2007; MSRG 2008]. PADRES is an open source 


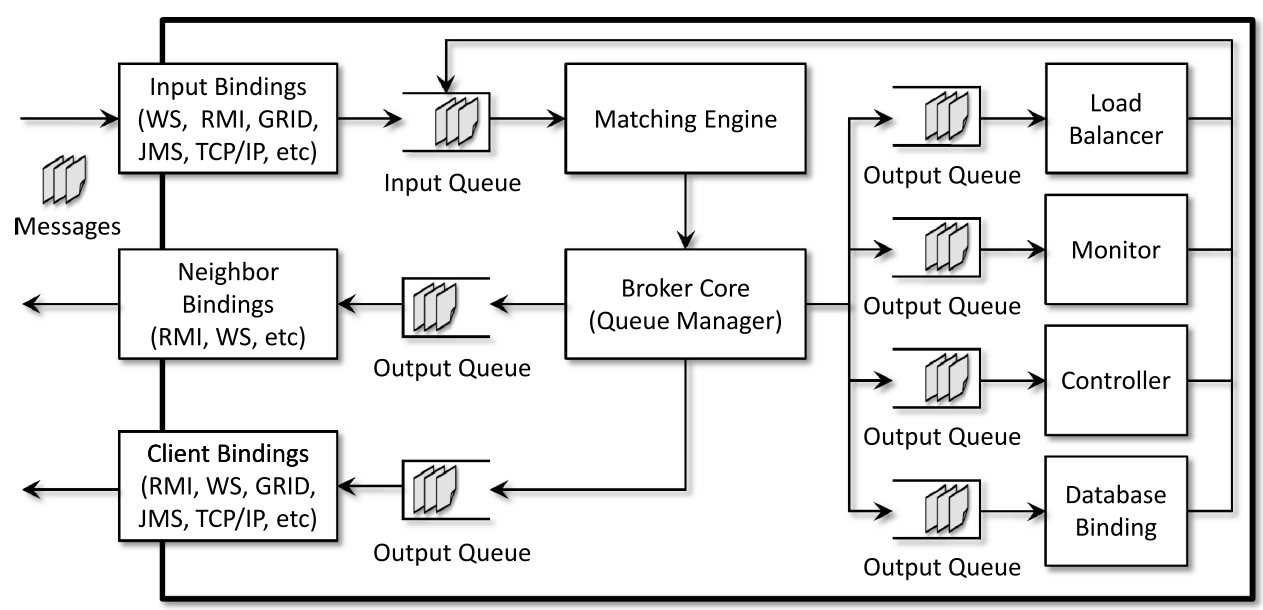

Fig. 10. Internal view of the PADRES broker.

distributed content-based publish/subscribe system developed by the Middleware Systems Research Group (MSRG) at the University of Toronto. The load balancer diagram previously shown in Figure 4 is integrated into the PADRES broker as the Load Balancer component illustrated in Figure 10. Using the flow of a message as a guide to describe the broker's architecture, a message from a neighbor broker or client comes into the broker through an input binding. The message is enqueued into the broker's input queue to wait for processing. The matching engine dequeues messages one by one from the input queue to perform the publish/subscribe match operation. Output from the matching engine is a set of messages with preset next hops, which can be a neighbor, a client, or one of the broker's internal modules. Each module in the PADRES broker has an associated output queue to receive messages from the matching engine. Messages sent by a module are enqueued back into the broker's input queue to let the matching engine decide how to route those messages. Each broker that is neighbor to this broker has an associated output queue to receive messages from this broker and a neighbor binding to transmit messages from this broker's output queue to the neighbor. Each client is attached to one of the broker's client bindings to receive messages from the broker. The purpose of bindings is to allow the broker to support multiple network communication protocols.

In the simulation mode of PADRES, network connections between brokers and clients are implemented as direct function calls. Hence, to get network performance measurements, bandwidth delays are simulated. Input and output queuing delays are simulated on the broker-level as brokers have input and output queues. Any other delays beneath the broker overlay, such as network latency, are not simulated because they are not optimized by the load balancing algorithm and hence have no effect other than being shown as extra overhead in the experimental results. Matching delays and matching engine memory consumptions are modeled as linear functions because each of these measurements cannot be isolated per broker instance within the JVM. 


\subsection{Experimental Setup}

We ran experiments on a cluster testbed and PlanetLab to evaluate our load balancing algorithm under real-world networking conditions and compare the results with those obtained previously from simulation. The cluster testbed we use consists of 22 identical machines with 4-GB memory, Intel Xeon 1.86$\mathrm{GHz}$ dual core CPUs, and 1 Gbps network links. On PlanetLab, random nodes around the world are selected. Different experiments on PlanetLab may use a different set of nodes because nodes unpredictably become either extremely slow or unreachable.

Deployments on both the cluster and PlanetLab are aided by the use of a tool developed by the MSRG called PANDA (Padres Automated Node Deployer and Administrator). This tool allows us to specify the experiment setup within a text formatted topology file such as the time and nodes to run brokers and clients, as well as any process specific runtime parameters such as the publication rate and stock quote publications for the publisher, subscription for the subscriber, neighbors for brokers, etc. The topology file is fed into PANDA, which then deploys the processes automatically. Deployments in the simulator do not use PANDA. Rather, the simulator itself allows one to specify a set of brokers with specific resource capacities, and a set of publishers and subscribers with their stockquote publications and subscriptions, respectively. Brokers can have custom CPU speeds, memory sizes, and network bandwidths. CPU speed affects the matching delay, and hence the input capacity of brokers. CPU speeds do not have any units as they are used for relativity sake, although they can be interpreted as $\mathrm{MHz}$. Memory sizes limit the amount of memory used by the matching engine and all message queues of a broker. Network bandwidth affects how fast messages can be sent.

On both real platforms, each broker process runs on a separate machine, while all publisher and subscriber processes run on the same machine to accurately measure end-to-end publication delivery delay. In simulation mode, all brokers and clients are instantiated within the same Java Virtual Machine (JVM). Brokers and overlay links are verified to be up and running before clients are deployed. In this work, we conducted experiments under three unique scenarios with both the random and proposed offload algorithms:

(1) Heterogeneous environment on PlanetLab;

(2) Heterogeneous environment through bandwidth throttling and matching delay scaling on a cluster testbed;

(3) Homogeneous environment on a cluster testbed.

These conditions range from a heterogeneous environment with unstable resources to a purely homogeneous environment with minimal outside disturbance. We also draw a comparison to show how our real-world findings are inline with our previous simulation results from Cheung and Jacobsen [2006].

As shown in Table III, default values for the load balancing parameters are used unless otherwise specified. Publishers on creation are assigned to publish stock quote publications of a particular company at a defined rate. Publishers 
Table III. Default Values of Load Balancing Parameters Used in Experiments

\begin{tabular}{|l|c|}
\hline Parameter (reference) & Value \\
\hline Local PIE publishing period (Section 3.3.2) & $30 \mathrm{~s}$ \\
Global PIE publishing period (Section 3.3.2) & $60 \mathrm{~s}$ \\
PIE ratio threshold (Section 3.3.2) & 0.025 \\
PIE delay threshold (Section 3.3.2) & 0.025 \\
Adaptive PIE history (Section 3.3.4) & 10 \\
Lower overload threshold (Section 3.3.3) & 0.9 \\
Higher overload threshold (Section 3.3.3) & 0.95 \\
Local ratio triggering threshold (Section 3.3.3) & 0.1 \\
Local delay triggering threshold (Section 3.3.3) & 0.1 \\
Global ratio triggering threshold (Section 3.3.3) & 0.15 \\
Global delay triggering threshold (Section 3.3.3) & 0.15 \\
Delay normalization factor (Section 3.3.3) & $0.1 \mathrm{~s}$ \\
Stabilize duration (Section 3.3.3) & $30 \mathrm{~s}$ \\
Stabilize percentage (Section 3.3.3) & 0.05 \\
Local detection minimum interval (Section 3.3.3) & $20 \mathrm{~s}$ \\
Local detection maximum interval (Section 3.3.3) & $40 \mathrm{~s}$ \\
Global detection minimum interval (Section 3.3.3) & $50 \mathrm{~s}$ \\
Global detection maximum interval (Section 3.3.3) & $70 \mathrm{~s}$ \\
Global load balancing request limit (Section 3.3.3) & 3 \\
Migration timeout (Section 3.4) & $5 \mathrm{~s}$ \\
PRESS samples (Section 4.1) & 50 \\
PRESS timeout (Section 4.1) & $30 \mathrm{~s}$ \\
Balance threshold (Section 5.1) & 0.005 \\
\hline
\end{tabular}

can be configured to change publication rates at any point in time in the experiment. Stock quote publications are real-world values obtained from Yahoo! Finance containing a stock's daily closing prices. ${ }^{5}$ A typical publication looks like this:

$$
\begin{aligned}
& \text { [class, 'STOCK'] , [symbol, 'YHOO'] , [open, 25.25], [high , 43. 00] } \\
& \text { [low, 24.50], [close, 33.00], [volume, 17030000], [date, '12-Apr-96'] }
\end{aligned}
$$

Subscribers are assigned to a fixed subscription based on one of the templates with the probabilities shown below. SUB_SYMBOL is randomly chosen out of the known stock symbols, with SUB_HIGH, SUB_LOW, and SUB_VOLUME replaced by a randomly chosen value of the same attribute from the stock's publication set.

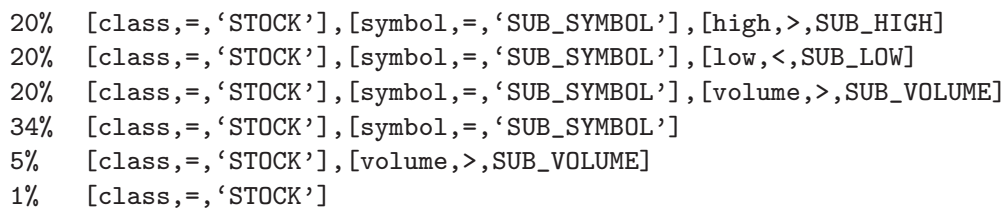

\subsection{Local Load Balancing Experiments}

In these experiments, we show how load and subscribers are distributed among the edge brokers in local load balancing. We also show the impact of the load balancing algorithm on the end-to-end delivery delay and amount of message

\footnotetext{
${ }^{5}$ The complete data set is available at http://www.msrg.org/datasets/acDataSet . 


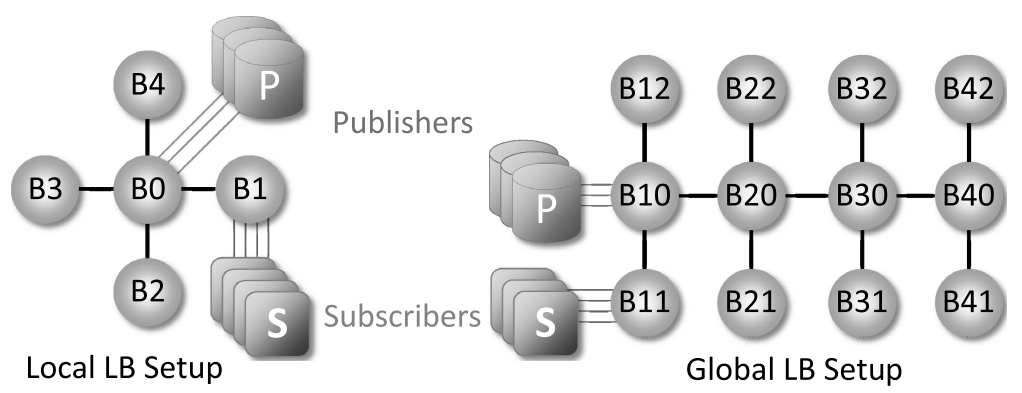

Fig. 11. Local (left) and global (right) load balancing experiment network topologies.

Table IV. Broker Specifications in Local Load Balancing Experiment

\begin{tabular}{|l|c|c|c|c|c|c|}
\hline \multirow{3}{*}{ Broker ID } & \multicolumn{3}{|c|}{ Simulation } & \multicolumn{3}{|c|}{ Heterogeneous Cluster } \\
\cline { 2 - 7 } & $\begin{array}{c}\text { CPU } \\
(\mathbf{M H z})\end{array}$ & $\begin{array}{c}\text { Memory } \\
(\text { MB })\end{array}$ & $\begin{array}{c}\text { Bandwidth } \\
(\text { Mbps })\end{array}$ & $\begin{array}{c}\text { Delay } \\
\text { Factor }\end{array}$ & $\begin{array}{c}\text { Memory } \\
(\text { MB })\end{array}$ & $\begin{array}{c}\text { Bandwidth } \\
(\text { Mbps })\end{array}$ \\
\hline B0 & 2000 & 32 & 10 & 0 & 2000 & 100 \\
B1 & 100 & 16 & 0.5 & $1 \times$ & 256 & 12 \\
B2 & 200 & 16 & 1 & $1.5 \times$ & 256 & 9 \\
B3 & 400 & 32 & 2 & $2.5 \times$ & 256 & 6 \\
B4 & 1000 & 32 & 5 & $10 \times$ & 256 & 0.85 \\
\hline
\end{tabular}

Table V. Client Specifications in Local Load Balancing Experiment

\begin{tabular}{|l|c|c|c|c|c|}
\hline Setup & $\begin{array}{c}\text { Number of } \\
\text { Publishers }\end{array}$ & $\begin{array}{c}\text { Avg Pub. } \\
\text { Rate } \\
\text { (msg/min) }\end{array}$ & $\begin{array}{c}\text { Time of } \\
+50 \% \text { Pub. } \\
\text { Rate }(\mathrm{s})\end{array}$ & $\begin{array}{c}\text { Number of } \\
\text { Subscribers }\end{array}$ & $\begin{array}{c}\text { O-Traffic } \\
\text { Subscribers } \\
\text { (\%) }\end{array}$ \\
\hline PlanetLab & 15 & 30 & 1000 & 120 & 50 \\
Hetero. Cluster & 60 & 30 & 1500 & 600 & 50 \\
Homo. Cluster & 60 & 30 & 1500 & 600 & 50 \\
Simulation & 40 & 30 & 3000 & 2000 & 25 \\
\hline
\end{tabular}

overhead introduced. Results on the scalability of the local load balancing algorithm and the accuracy of load estimations are also presented.

The setup used for the local load balancing experiment involves four edge brokers connected to one cluster-head broker to form a star topology as shown on the left of Figure 11. This setup mimics a cluster at one data center as found in practice. Specifications on both heterogeneous cluster and simulation setups for the brokers are summarized in Table IV and for clients in Table V. On experiment startup, brokers $B 0, B 1, B 2, B 3$, and $B 4$ are instantiated simultaneously. After all broker processes and links are established, all publishers connect to broker $B 0$. Immediately following, all subscribers connect to broker $B 1$ at a time chosen randomly within the first $500 \mathrm{~s}$ on PlanetLab, $600 \mathrm{~s}$ on the cluster testbed, and $1010 \mathrm{~s}$ in the simulator using a uniform random distribution. The greatest number of publishers and subscribers are chosen to the best of our ability without overloading the entire system. The reasoning behind connecting all subscribers at $B 1$ is to try overloading this broker and observe how the load balancing algorithm prevents overload by dynamically distributing the subscribers to other edge brokers. For the experiments on churn, subscribers join the system at a rate of 60 per minute continuously. 
Each subscriber remains connected to the system for 7 to 14 minutes (chosen randomly) until which the subscriber unsubscribes and disconnects from the broker. We experimented with two joining patterns: (1) grouped-random where all subscribers within each minute join the same random edge broker, and (2) individually-random where each subscriber joins a random edge broker. Of all subscribers, a certain percentage of them sink zero traffic, which means their subscriptions do not match any publications in the system. Sometime into the experiment (see Table V), 50\% of the publishers are randomly chosen to have their publication rates increased by $100 \%$. This shows the dynamic behavior of the load balancer under changing load conditions. For the experiment on edge broker scalability, all edge brokers are homogeneous except when running on PlanetLab.

6.3.1 Broker Load Distribution. Figure 12 shows the load distribution among the four edge brokers in local load balancing on the heterogeneous cluster, the homogeneous cluster, PlanetLab, and the simulator. Graphs for matching delay are not shown as their trend looks similar to the input utilization ratio graphs. Input utilization ratio graphs for the homogeneous cluster and PlanetLab are omitted because they closely resemble Figure 12(c) where their values never exceed the threshold to trigger load balancing, indicating that the output resource is the only bottleneck. Comparing the graphs for the three performance metrics on the heterogeneous cluster and simulation setups in Figure 12(a)-(d):

-Even though all subscribers join broker B1, those subscribers get offloaded to other edge brokers once a sizeable load difference or overload occurs.

-All performance metrics converge until the $50 \%$ increase publication rate disturbance.

-All performance metrics converge and stabilize within the configured thresholds.

The graphs from the simulations have less noise as there is absolutely no outside activity disturbances such as operating system background processes, user processes, and Java's garbage collector. The workload used in simulation have much more subscribers joining $B 1$ than the workload in real experiments, which causes $B 1$ to become temporarily overload within the first $1000 \mathrm{~s}$ in the simulation but not in the real experiment.

Figure 12(e) shows that the output utilization ratio does not converge on PlanetLab. This is due to the stability restriction of the output offload algorithm. Also worth noting is that load fluctuations are more pronounced on PlanetLab than on the cluster testbed. We believe this is due to the greater resource contention as a result of shared nodes among world wide users and added dynamics of the Internet links. Figure 12(f) shows that our proposed load balancing algorithm balances load effectively on a homogeneous platform as well.

In the presence of churn, the load balancing algorithm continues to operate in a stable manner while trying to keep the load among brokers balanced. 


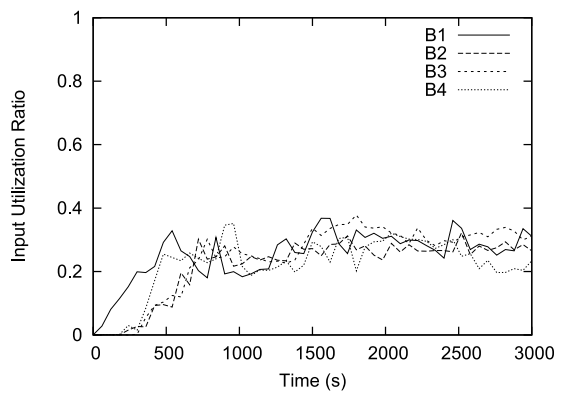

(a) Input util ratio on hetero. cluster

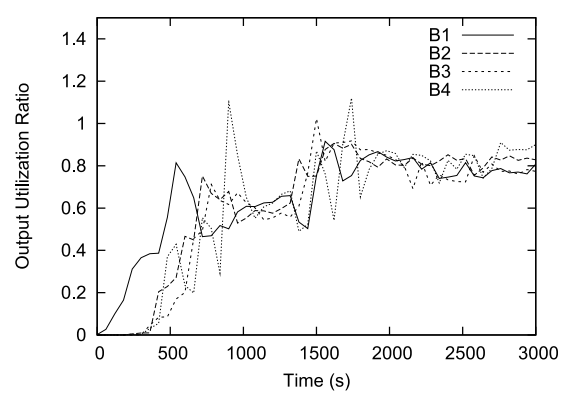

(c) Output util ratio on hetero. cluster

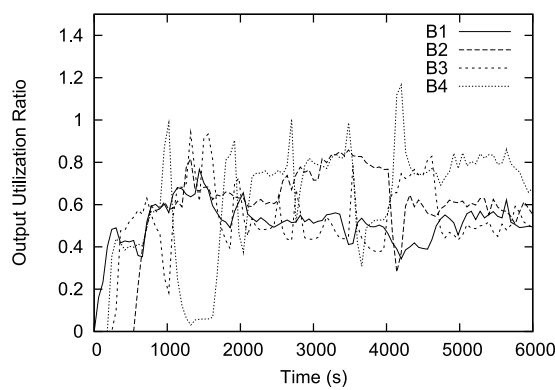

(e) Output util ratio on PlanetLab

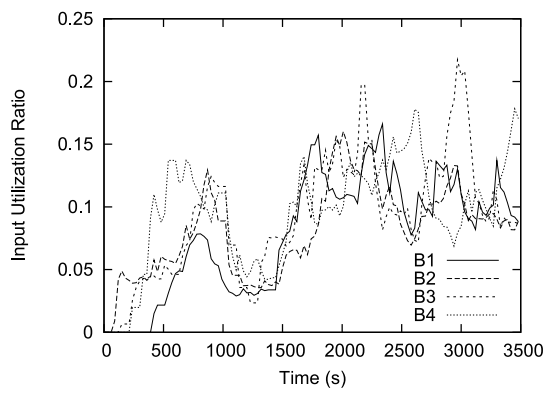

(g) Input util ratio on cluster (churn)

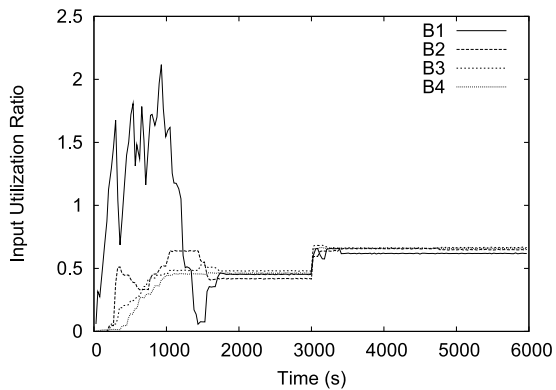

(b) Input util ratio in simulation

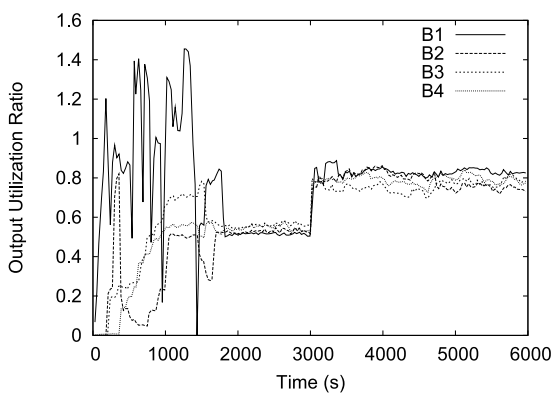

(d) Output util ratio in simulation

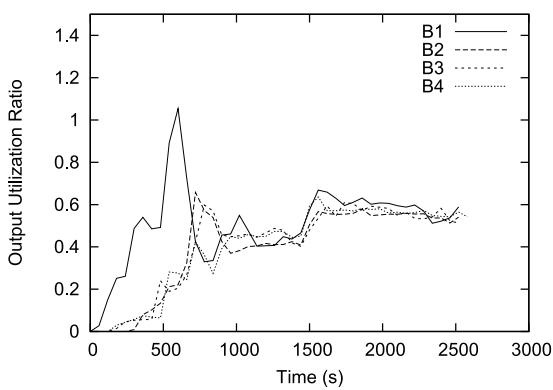

(f) Output util ratio on homo. cluster

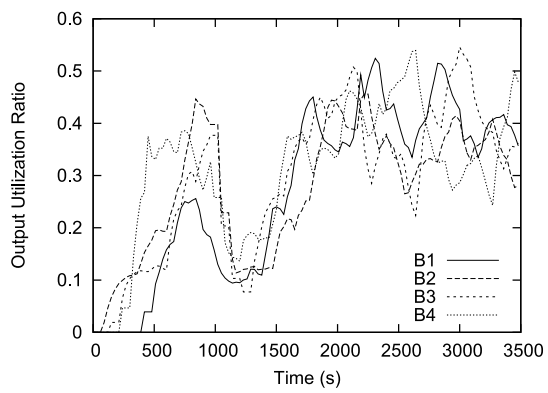

(h) Output util ratio on cluster (churn)

Fig. 12. Load distribution in local load balancing on various platforms. 


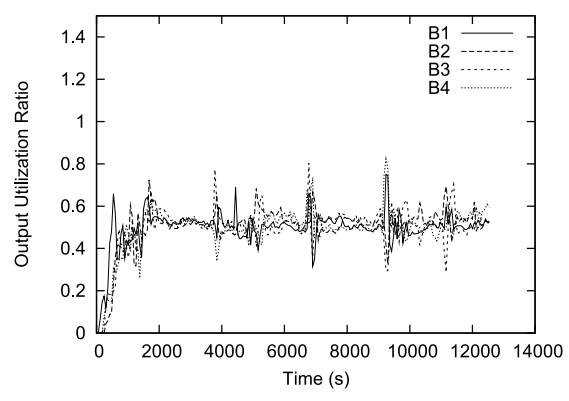

(a) Output util ratio on homo. cluster

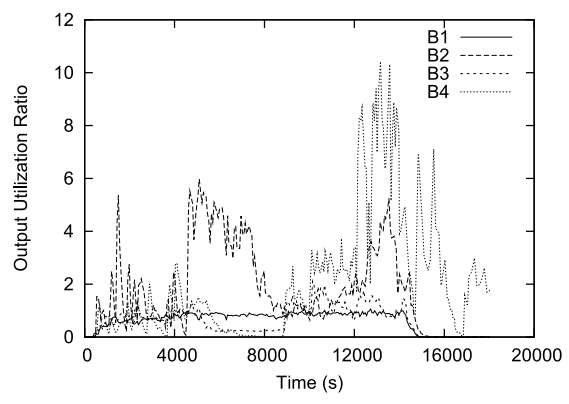

(b) Output util ratio on PlanetLab

Fig. 13. Output load distribution in local load balancing using the naive algorithm.

Results for grouped-random joins are shown in Figure 12(g) and 12(h), while the graphs for individually-random joins have the same trend but with load fluctuations of lesser magnitude. Though not shown, the naive approach performed similar to the proposed approach under churn on the homogeneous cluster testbed.

Figure 13(a) shows that the output load converges on the homogeneous setup when using the naive approach under the workload where all subscribers join broker B1. However, there are three significant differences with Figure 12(f). First, a heavy concentration of sharp spikes in the first $2000 \mathrm{~s}$ indicate that more offload sessions each involving a greater number of subscription swaps are needed to find a stable subscription distribution. This indicates that the naive algorithm is not effective in choosing subscriptions in balancing load, resulting in more offload sessions than is necessary, which in turn leads to inefficiency. Second, due to the unpredictable trial-and-error offload sessions, the naive algorithm takes $500 \mathrm{~s}$ longer to converge than the proposed approach. Third, intermittent small noise disturbances trigger unnecessary large offloads that result in large spikes in load. On both PlanetLab (Figure 13(b)) and the heterogeneous cluster setup (not shown), the naive approach actually causes the output utilization ratio on brokers $B 2, B 3$, and $B 4$ to diverge, creating system-wide overload.

In short, our results on the cluster testbed and PlanetLab show that local load balancing is effective on both homogeneous and heterogeneous environments. As well, a naive offload algorithm that has no subscription load and space awareness is unable to load balance effectively and efficiently in a content-based publish/subscribe system.

6.3.2 Client Perceived Delivery Delay. Figure 14 shows the average end-toend delivery delay on all testbeds. On both the cluster and PlanetLab, the load balancing algorithm prevents overload from occurring at $B 1$ by dynamically distributing incoming subscribers to other edge brokers. As a result, the delivery delay stays constant at around $0.2 \mathrm{~s}$ throughout the experiment rather than increase without bound as in an overload condition. Two large spikes in both graphs are due to a slight period of output overload at broker B4 in both cases.

ACM Transactions on Computer Systems, Vol. 28, No. 4, Article 9, Pub. date: December 2010. 


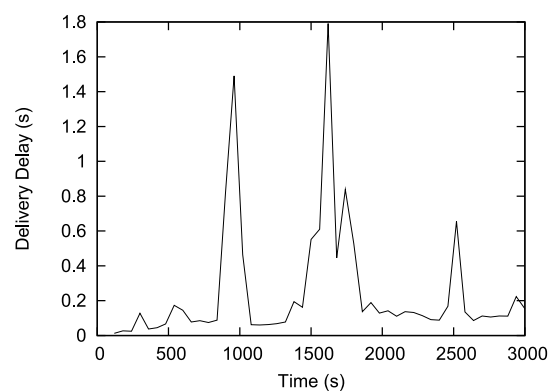

(a) Heterogeneous cluster

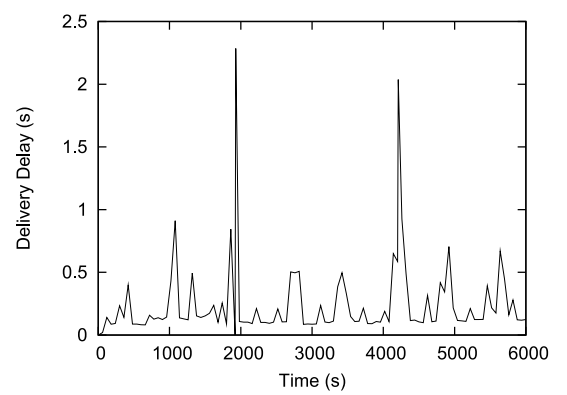

(c) PlanetLab

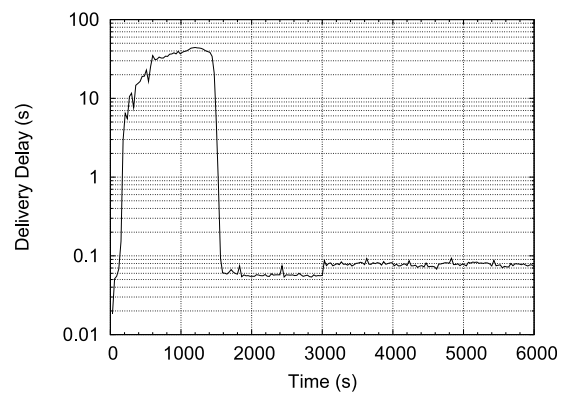

(b) Simulation

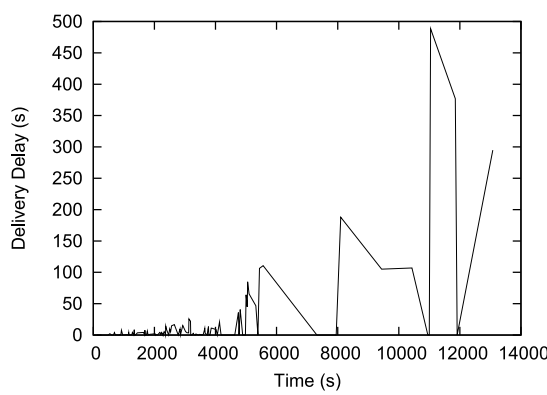

(d) Naive Alg. on PlanetLab

Fig. 14. Average end-to-end delivery delay in local load balancing on various setups.

In the presence of churn, the delivery delay remained constant and never exceeded $0.1 \mathrm{~s}$. If the rate of subscriber joins far exceeds the offloading rate, as shown in Figure 14(b), then the delivery delay grows until the broker is no longer overloaded and processes all messages in its queues. With the naive approach running on PlanetLab as shown in Figure 14(d), not only does overload yield high delivery delays but also lead to zero message delivery as brokers behave erratically from insufficient memory at $5000 \mathrm{~s}$ and finally crash at $13000 \mathrm{~s}$. In summary, the proposed load balancing algorithm is able to minimize disruptions to the delivery delay by preventing overload in the system.

6.3.3 Subscriber Distribution Among Brokers. Figure 15(a) and 15(c) show that the load balancing algorithm can account for heterogeneous brokers by assigning more subscribers to more powerful brokers. Figure 15(d) shows that the naive algorithm needs to offload a much larger number of subscribers and perform many trial and error offload sessions before converging to a stable state. This is because the algorithm has no sense of the traffic associated with each subscription. Even for slight load differences, the naive algorithm swaps as much as hundreds of subscribers whereas the proposed algorithm offloads less than ten even under unstable conditions such as on PlanetLab (Figure 15(c)).

6.3.4 Load Balancing Message Overhead. Figure 16(a) and 16(b) show that message overhead, which includes subscription messages and PIE 


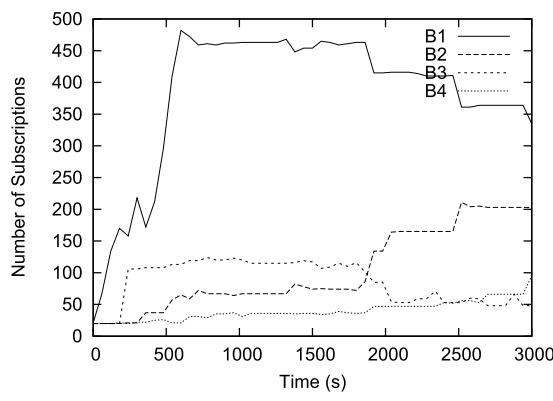

(a) Heterogeneous cluster

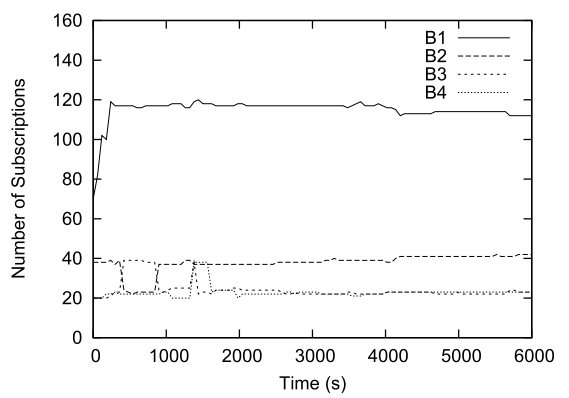

(c) PlanetLab

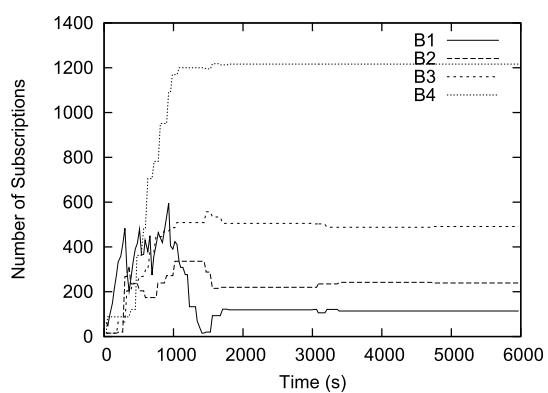

(b) Simulation

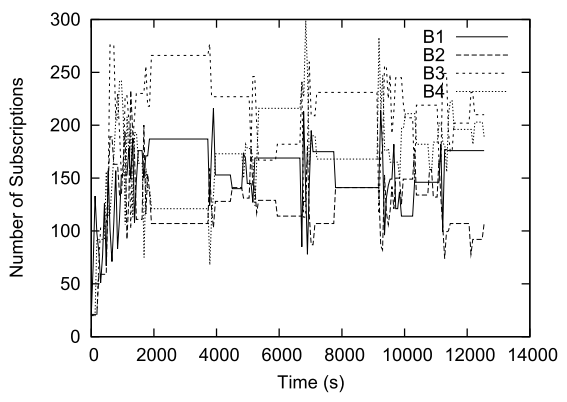

(d) Random on homogeneous cluster

Fig. 15. Subscriber distribution in local load balancing on various setups.

messages, across all brokers is less than 50\% during subscriber joins and less than $0.2 \%$ and $1.1 \%$ after load balancing has converged in simulation and real experiments, respectively. If we examine the overhead message rates with conventional and Adaptive PIE as is shown in Figure 16(c) and 16(d), respectively, the highest source of message overhead is the migrating subscribers' subscriptions. While load balancing, there are $0.4 \mathrm{msg} / \mathrm{s}$ of load balancing coordination messages, which consist of load balancing request and reply and subscriber migration coordination messages, and $0.85 \mathrm{msg} / \mathrm{s}$ of PIE messages. With Adaptive PIE however, there are $40 \%$ less PIE messages. Once the system stabilizes with no load balancing activity, there are no load balancing coordination messages and Adaptive PIE reduces the amount of PIE messages by $65 \%$ compared to conventional PIE. In the churn experiments where the load of brokers constantly fluctuates, Adaptive PIE reduces the amount of PIE overhead messages, including PIE advertisements, subscriptions, and unsubscriptions, by up to $30 \%$. In summary, the added cost of running the load balancing algorithm is almost negligible and does not hinder the scalability of the existing publish/subscribe system.

6.3.5 Edge Broker Scalability. To measure how the publish/subscribe system scales with the number of brokers in a cluster, we focus on the load reduced on each edge broker as well as any gains in delivery delay. Figure 17(a) and 17 (b) indicate that the output utilization ratio, which is the limiting resource on both cluster and Planetlab setups, is reduced as the number of edge 


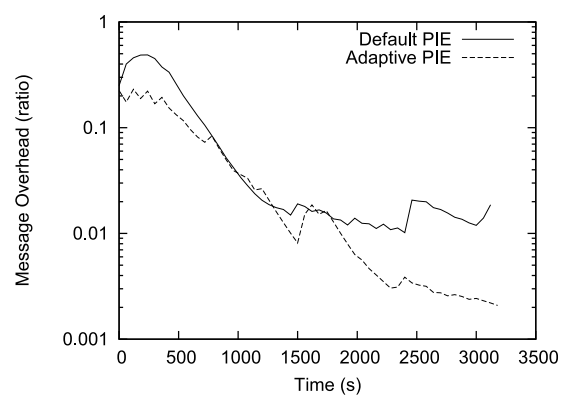

(a) Heterogeneous cluster

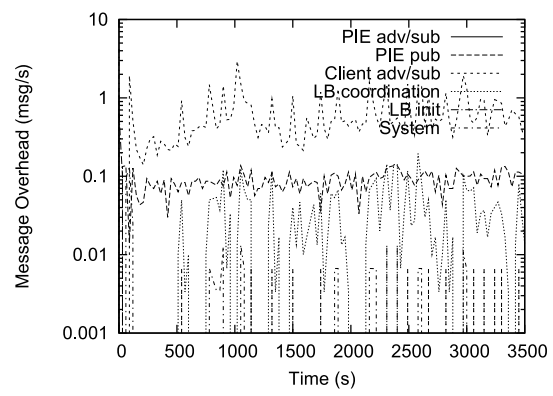

(c) Homo. cluster with default PIE (churn)

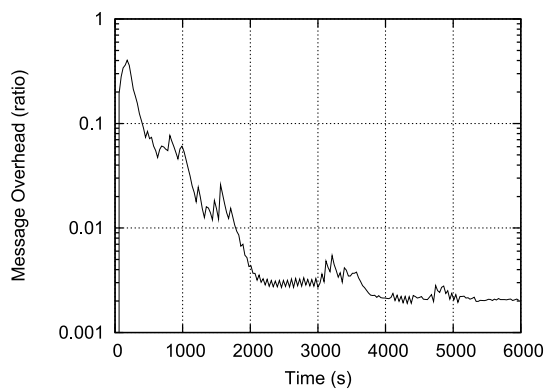

(b) Simulation

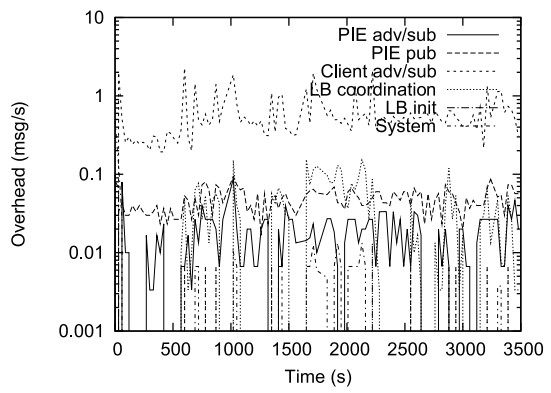

(d) Homo. cluster with Adap. PIE (churn)

Fig. 16. Message overhead in local load balancing.

brokers increase. This indicates that the proposed load balancing algorithm indeed allows the system to scale with the number of brokers. However, gains in delivery delay are insignificant past adding five edge brokers as witnessed on the cluster testbed and simulation. On PlanetLab, where Internet link delays dominate over broker processing delay, there is no observable gain in delivery delay.

6.3.6 Load Estimation Accuracy. Load estimation accuracy determines the effectiveness of each local load balancing session. Figure 18 shows the estimation accuracy for all performance metrics on the heterogeneous cluster setup and the input utilization ratio obtained from simulation. Graphs for the matching delay and output utilization ratio estimation in the simulation are not shown here because they have the same trends and exception cases as the input utilization ratio. Dots on the $y=x$ line denote $100 \%$ accuracy. In the experiment on the cluster testbed, a majority of the data points lie close to the $y=x$ line as shown in Figure 18(a)-(c). Specifically, the mean average percentage error of both input and output utilization ratios is $17 \%$, and matching delay is $13 \%$. Compared to the simulation, the input and output utilizations are $8 \%$ and $3 \%$, respectively, while there is no error in matching delay estimation as matching delays are simulated by a predefined linear function. The amount of error represented in the simulation results indicates the prediction error of future traffic based on the present data. We believe the additional error in the real experiments are due to external disturbances such as CPU and network 


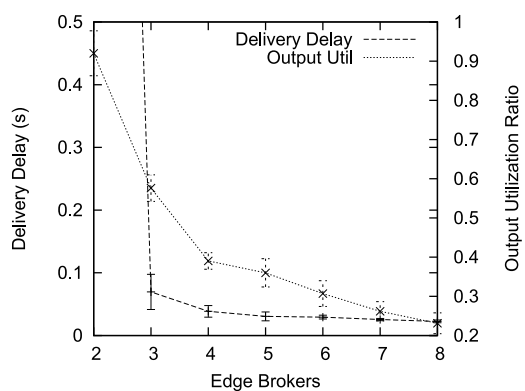

(a) Homogeneous cluster

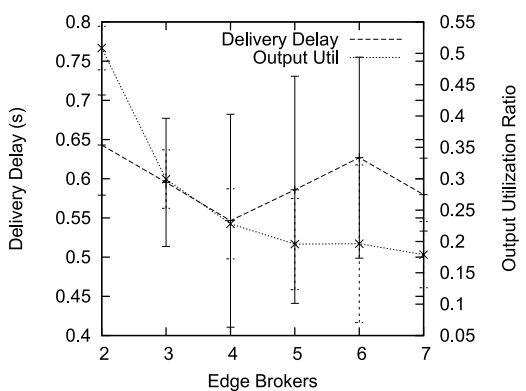

(b) PlanetLab

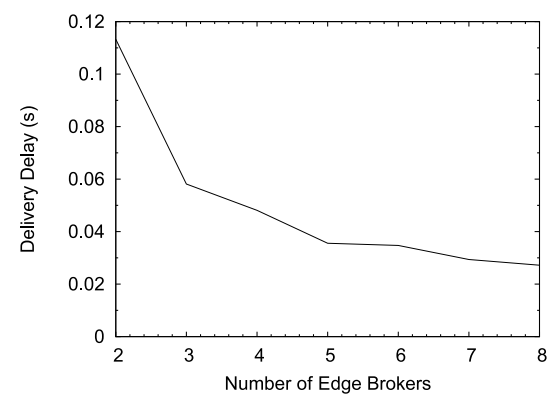

(c) Simulation

Fig. 17. System scalability by adding more edge brokers into a cluster.

contention on the shared cluster nodes, interruptions in processing from Java's garbage collector, estimation of number of bytes transmitted over RMI by taking the length of serialized message objects, and modeling matching delay with a linear function. With $90 \%$ of load estimations having a mean average percentage error of less than $11 \%$ on PlanetLab, this means that at least $90 \%$ of local load balancing sessions are effective, that is, they do not need a followup session to further balance load. Followup load balancing sessions slow down the overall response time of the algorithm and increase overhead compared to a single effective load balancing session.

\subsection{Global Load Balancing}

In these experiments, we will show how global load balancing sessions are coordinated and how load is transferred from one cluster to another in global load balancing. We also show the scalability of global load balancing by scaling up the number of clusters in the system.

The setup used for the global load balancing experiment involves 12 brokers organized into 4 clusters, with 2 edge brokers per cluster as shown on the right of Figure 11. This setup mimics multiple data centers (clusters) connected together to form the complete publish/subscribe system [Cooper et al. 2008; Reumann 2009]. Specifications on both heterogeneous cluster and simulation setups regarding the brokers are summarized in Table VI. Client configurations are the same as in the local load balancing setup as shown in Table V 


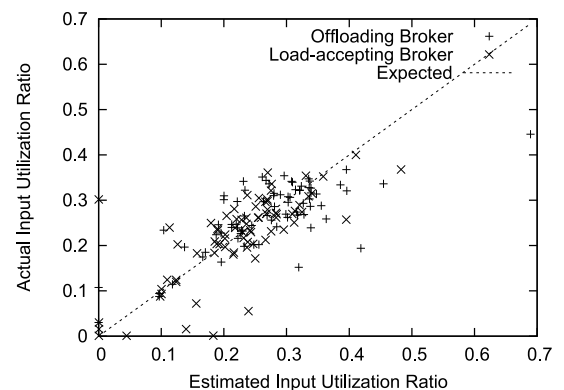

(a) Input util ratio on cluster

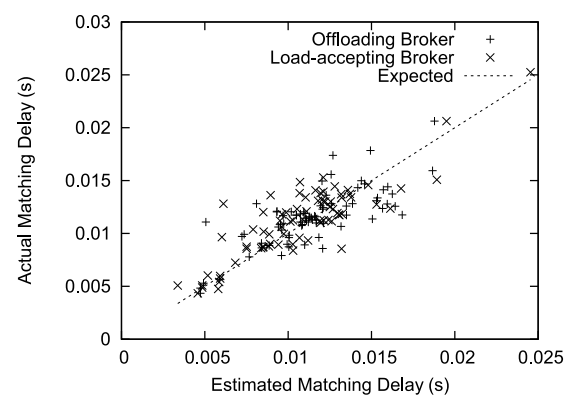

(c) Matching delay in cluster

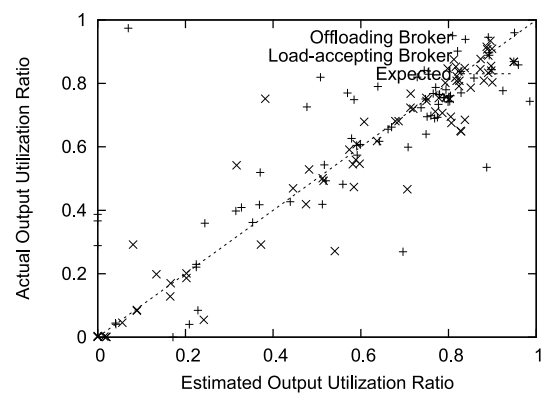

(b) Output util ratio on cluster

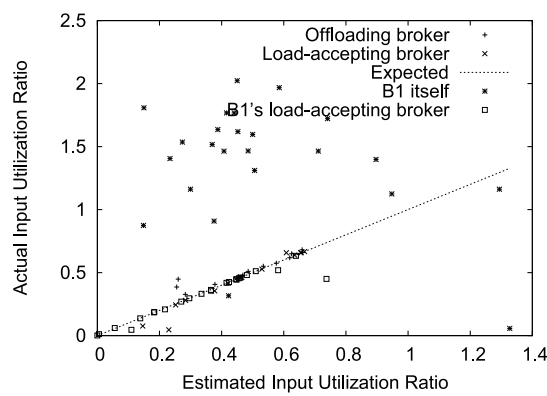

(d) Input util ratio in simulation

Fig. 18. Load estimation accuracy on heterogeneous cluster testbed and simulation.

Table VI. Broker Specifications in Global Load Balancing Experiment

\begin{tabular}{|l|c|c|c|c|c|c|}
\hline \multirow{3}{*}{ Broker ID } & \multicolumn{3}{|c|}{ Simulation } & \multicolumn{3}{|c|}{ Heterogeneous Cluster } \\
\cline { 2 - 7 } & $\begin{array}{c}\text { CPU } \\
(\mathrm{MHz})\end{array}$ & $\begin{array}{c}\text { Memory } \\
(\mathrm{MB})\end{array}$ & $\begin{array}{c}\text { Bandwidth } \\
(\text { Mbps })\end{array}$ & $\begin{array}{c}\text { Delay } \\
\text { Factor }\end{array}$ & $\begin{array}{c}\text { Memory } \\
(\text { MB })\end{array}$ & $\begin{array}{c}\text { Bandwidth } \\
(\text { Mbps })\end{array}$ \\
\hline B11 & 300 & 128 & 1 & $1 \times$ & 2000 & 10 \\
B12 & 200 & 128 & 0.5 & $0.5 \times$ & 512 & 12 \\
B21 & 350 & 64 & 1 & $1.0 \times$ & 512 & 10 \\
B22 & 400 & 64 & 1.5 & $1.5 \times$ & 512 & 8 \\
B31 & 266 & 64 & 1 & $2.5 \times$ & 512 & 6 \\
B32 & 700 & 64 & 2 & $1.5 \times$ & 512 & 9 \\
B41 & 166 & 64 & 0.5 & $1 \times$ & 512 & 8 \\
B42 & 233 & 128 & 0.5 & $3 \times$ & 512 & 5 \\
\hline
\end{tabular}

except that half the publishers in the simulation increase their publication rate by $100 \%$ at $8000 \mathrm{~s}$. On experiment startup, all brokers are instantiated simultaneously. After all broker processes and links are established, all publishers connect to broker B10. Immediately following, all subscribers connect to broker $B 11$ at a time chosen randomly within the first $500 \mathrm{~s}$ on PlanetLab, $600 \mathrm{~s}$ on the cluster testbed, and $1010 \mathrm{~s}$ in the simulation using a uniform random distribution. The experiment on churn uses the same join rates, durations, and patterns as described in Section 6.3. The greatest number of publishers and subscribers are chosen to the best of our ability without overloading the entire system. For the experiment on the cluster scalability, there are two edge 


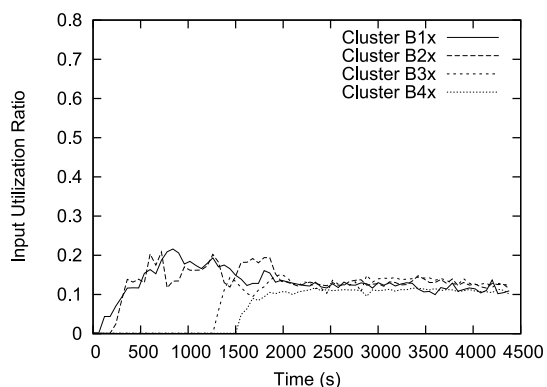

(a) Input util ratio on cluster testbed

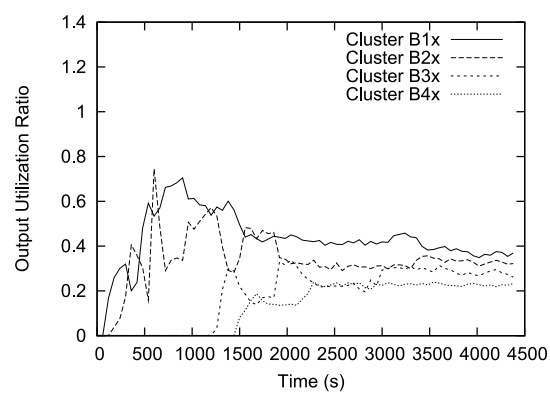

(c) Output util ratio on cluster testbed

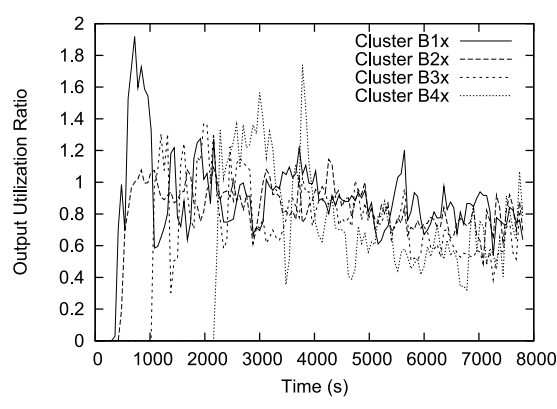

(e) Output util ratio on PlanetLab

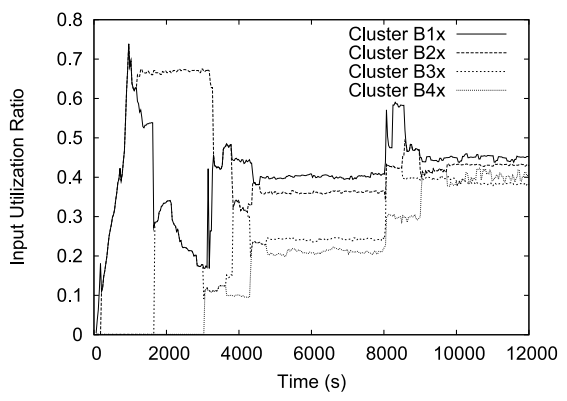

(b) Input util ratio in simulation

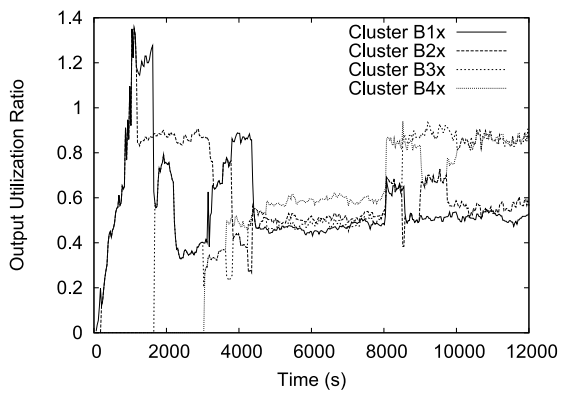

(d) Output util ratio in simulation

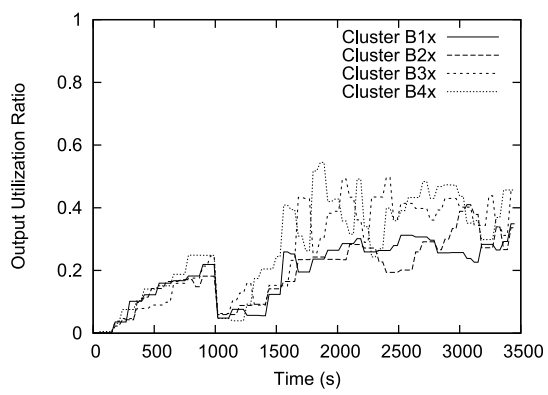

(f) Output util ratio on cluster (churn)

Fig. 19. Load distribution among clusters in global load balancing.

brokers in each cluster, with all edge brokers having equal resource capacity except on PlanetLab.

6.4.1 Cluster Load Distribution. The average load of each cluster engaged in global load balancing is shown in Figure 19. Figure 19(a) and 19(c) show the results of running on the heterogeneous cluster testbed setup, while 19(b) and 19(d) show the results of running on the simulator. On PlanetLab, only the output utilization ratio graph is shown (Figure 19(e)) because only the output resource is constrained enough to trigger load balancing. Whenever a cluster performs global load balancing with another cluster, the two clusters' loads appear merged on the graph. Compared to local load balancing, global load balancing takes longer to converge because the clusters are arranged in ACM Transactions on Computer Systems, Vol. 28, No. 4, Article 9, Pub. date: December 2010. 


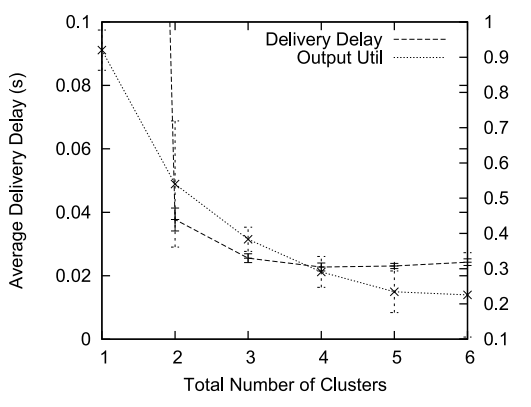

(a) Homogeneous cluster testbed

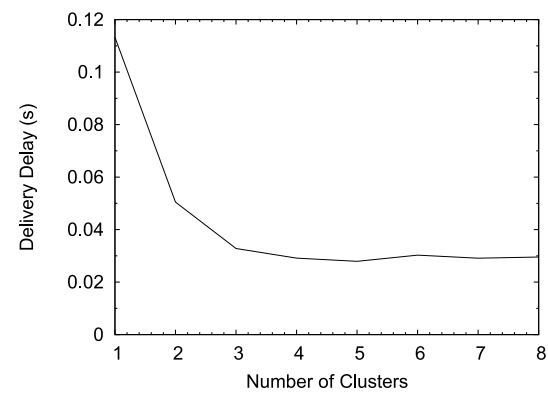

(b) Simulation

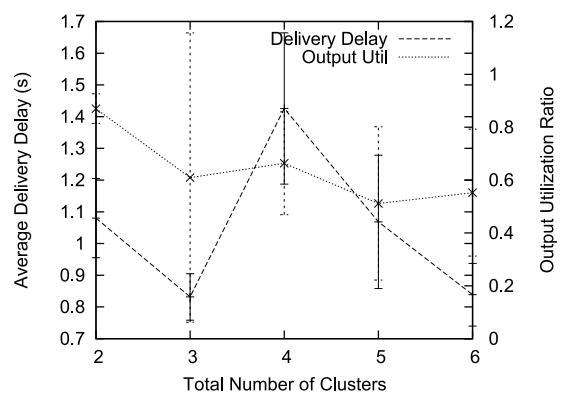

(c) PlanetLab

Fig. 20. System scalability by adding more clusters.

a chain topology in this experiment, which limits parallelizing load balancing sessions. For example, when cluster $B 2 x$ load balances with $B 3 x$, cluster $B 1 x$ stays idle. Global load balancing converges $2000 \mathrm{~s}$ after the load balancing algorithm starts distributing load to the last cluster $(B 4 x)$ on all setups. When global load balancing converges, clusters further away from cluster B1x carries a smaller amount of load. This is desired so that subscribers do no get relocated too far into the network away from the publisher. Figure 19(e) shows that the load balancing algorithm works even in the presence of system overload until load is evenly distributed among all clusters to stop overload. Under churn conditions, both the proposed (Figure 19(f)) and naive approaches continually perform load balancing without diverging. In summary, global load balancing is able to effectively distribute load originating from one broker to others brokers in surrounding clusters.

6.4.2 Cluster Scalability. When clusters are organized in a chain-like topology, there is a load diminishing effect on clusters further away from the source of load, namely cluster B1x in our experiment. This is primarily due to the global detection threshold setting where clusters only engage in load balancing if one of their performance metrics exceeds a certain value. On both the cluster testbed (Figure 20(a)) and in simulations (Figure 20(b)), clusters more than 3 cluster-hops away from cluster $B 1 x$ no longer reduce the overall delivery delay. Instead, the delivery delay gradually increases as the publications 
travel over more broker hops to reach the subscribers. This indicates a tradeoff between load distribution and client locality. Perfect load distribution onto all nodes will result in a more stable system, but because the clients are scattered throughout the entire network, end-to-end delivery delay is sacrificed. However, this trend is not observable on PlanetLab (Figure 20(c)) because the magnitude of delivery delay is dominated by Internet link delays, which heavily depend on the distance between the brokers and subscribers in the world. Yet, on both real life platforms, the overall system output utilization ratio decreases with additional clusters.

\section{CONCLUSIONS}

In this article, we presented a load balancing solution with four main contributions: a load balancing framework, load estimation methodologies, three offload algorithms, and experimental results that demonstrate the performance and effectiveness of our approach. The load balancing framework consists of the PEER architecture, a distributed load exchange protocol called PIE, and detection and mediation mechanisms at the local and global load balancing levels. The core of the load estimation is PRESS, which uses an efficient bit vector approach to estimate the input and output publication loads of a subscription. Each of the three offload algorithms are designed to load balance on a particular performance metric with minimal side-effects and proven stability. Both the load estimation and offload algorithms are independent of the load balancing framework. Our solution inherits all of the most desirable properties that make a load balancing algorithm flexible. PIE contributes to the distributed and dynamic nature of our load balancing solution by allowing each broker to invoke load balancing whenever necessary. Adaptiveness is provided by the three offload algorithms that load balance on a unique performance metric. The local mediator gives transparency to the subscribers throughout the offload process. Finally, load estimation with PRESS allows the offload algorithms to account for broker and subscription heterogeneity. A number of unique publish/subscribe concepts were introduced and put into use, such as the use of publication messages for control messaging, TTL to limit the propagation diameter of load information messages, prioritizing control messages to preserve load balancer response times under overload conditions, message payloads to carry matching-independent data, and the local subscribe operation utilized by PRESS to dynamically monitor the amount of shared traffic between the offloading and load-accepting brokers. All of our ideas presented in this article are prototyped and tested on the open-source publish/subscribe system called PADRES [MSRG 2008].

Results from PlanetLab, a cluster testbed, and the simulator show that our load balancing algorithm prevents overload by distributing subscribers while simultaneously balancing three performance metrics among edge brokers. Our algorithm enables the publish/subscribe system to scale with the amount of resources available to it and is effective in both homogeneous and heterogeneous environments. By adaptively subscribing to load information, message overhead of the load balancing infrastructure is reduced by an additional $65 \%$ to 
$0.2 \%$ in our experiments on the cluster testbed. Our results also show that a naive load balancing solution that cannot identify subscription space and load is not only inefficient but can also lead to system instability.

\section{ACKNOWLEDGMENTS}

We would like to sincerely thank Serge Mankovskii, CA Technologies, Inc. for his helpful guidance and thoughtful comments throughout the PADRES project. Our gratitude also goes to the long list of PADRES project alumni for their support and assistance throughput this research.

\section{REFERENCES}

Adler, M., Ge, Z., Kurose, J. F., Towsley, D., And Zabele, S. 2001. Channelization problem in large scale data dissemination. In Proceedings of the International Conference on Network Protocols (ICNP'01). IEEE Computer Society, Los Alamitos, CA, 100-109.

Aekaterinidis, I. AND TRIANTAFILlou, P. 2006. PastryStrings: A comprehensive content-based publish/subscribe DHT network. In Proceedings of the IEEE International Conference on Distributed Computing Systems (ICDCS'06). IEEE Computer Society, Los Alamitos, CA, 23-42.

Aleksy, M., Korthaus, A., AND Schader, M. 2001. Design and implementation of a flexible load balancing service for CORBA-based applications. In Proceedings of the International Conference on Parallel and Distributed Processing Techniques and Applications (PDPTA'01). IEEE Computer Society, Los Alamitos, CA.

Altinel, M. AND FrankLin, M. J. 2000. Efficient filtering of XML documents for selective dissemination of information. In Proceedings of the International Conference on Very Large Data Bases (VLDB'00). Morgan-Kaufmann Publishers Inc., San Francisco, CA, 53-64.

BAldoni, R., MARChetti, C., Virgillito, A., AND Vitenberg, R. 2005. Content-based publishsubscribe over structured overlay networks. In Proceedings of the IEEE International Conference on Distributed Computing Systems (ICDCS'05). IEEE Computer Society, Los Alamitos, CA, 437-446.

Baldoni, R., Beraldi, R., Quema, V., Querzoni, L., And Tucci-Piergiovanni, S. 2007. TERA: Topic-based event routing for peer-to-peer architectures. In Proceedings of the Inaugural International Conference on Distributed Event-Based Systems (DEBS'07). ACM, New York, NY, $2-13$.

Banavar, G., Chandra, T., Mukherjee, B., Nagarajarao, J., Strom, R. E., and Sturman, D. C. 1999. An efficient multicast protocol for content-based publish-subscribe systems. In Proceedings of the IEEE International Conference on Distributed Computing Systems (ICDCS'99). IEEE Computer Society, Los Alamitos, CA, 262-272.

Barth, T., Flender, G., Freisleben, B., AND Thilo, F. 1999. Load distribution in a CORBA environment. In Proceedings of the International Symposium on Distributed Objects and Applications (DOA'99). IEEE Computer Society, Los Alamitos, CA, 158-166.

BERMAN, F. AND WOLSKI, R. 1996. Scheduling from the perspective of the application. In Proceedings of the IEEE International Symposium on High Performance Distributed Computing (HPDC'96). IEEE Computer Society, Los Alamitos, CA, 100-111.

Bharambe, A. R., RAO, S., AND Seshan, S. 2002. Mercury: A scalable publish-subscribe system for internet games. In Proceedings of the Workshop on Network and System Support for Games (NetGames'02). ACM, New York, NY, 3-9.

CAO, F. AND SinGH, J. P. 2004. Efficient event routing in content-based publish-subscribe service networks. In Proceedings of the IEEE International Conference on Computer Communications (INFOCOM'04). 924-940.

Cardellini, V., Colajanni, M., AND Yu, P. S. 1999a. DNS dispatching algorithms with state estimators for scalable web-server clusters. World Wide Web 2, 3, 101-113.

Cardellini, V., Colajanni, M., And Yu, P. S. 1999b. Dynamic load balancing on web-server systems. IEEE Internet Comput. 3, 3, 28-39. 
Carzaniga, A., Rosenblum, D. S., And Wolf, A. L. 2001. Design and evaluation of a wide-area event notification service. ACM Trans. Comput. Syst. 19, 3, 332-383.

Casalicchio, E. And Morabito, F. 2007. Distributed subscriptions clustering with limited knowledge sharing for content-based publish/subscribe systems. In Proceedings of the IEEE International Symposium on Network Computing and Applications. 105-112.

Castelli, S., Costa, P., AND Picco, G. P. 2008. HyperCBR: Large-scale content-based routing in a multidimensional space. In Proceedings of the IEEE International Conference on Computer Communications (INFOCOM'08). 1714-1722.

Castro, M., Druschel, P., Kermarrec, A.-M., and Rowstron, A. 2002. SCRIBE: A large-scale and decentralized application-level multicast infrastructure. IEEE J. Select. Areas Comm. 20, 1489-1499.

Chand, R. AND Felber, P. 2005. Semantic peer-to-peer overlays for publish/subscribe networks. In Proceedings of Euro-Par Parallel Processing. Vol. 3648. 1194-1204.

Chen, C., Jacobsen, H.-A., And Vitenberg, R. 2010. Divide and conquer algorithms for publish/subscribe overlay design. In Proceedings of the International Conference on Distributed Computing Systems (ICDCS'10). IEEE Computer Society, Los Alamitos, CA, 622-633.

Chen, Y. AND SchwAN, K. 2005. Opportunistic overlays: Efficient content delivery in mobile ad hoc networks. In Proceedings of the ACM/IFIP/USENIX International Conference on Middleware (Middleware'05). Springer-Verlag, Berlin, 354-374.

Cheung, A. K. Y. 2006. Dynamic load balancing in distributed content-based publish/subscribe. M.A.Sc. dissertation. University of Toronto.

Cheung, A. K. Y. And Jacobsen, H.-A. 2006. Dynamic load balancing in distributed contentbased publish/subscribe. In Proceedings of the ACM /IFIP/USENIX International Conference on Middleware (Middleware'06). Springer-Verlag, Berlin, 141-161.

Cooper, B. F., Ramakrishnan, R., Srivastava, U., Silberstein, A., Bohannon, P., Jacobsen, H.-A., PuZ, N., Weaver, D., AND Yerneni, R. 2008. PNUTS: Yahoo!'s hosted data serving platform. Proc. VLDB Endow. 1, 2, 1277-1288.

Cugola, G., Di NitTo, E., And Fuggetta, A. 2001. The JEDI event-based infrastructure and its application to the development of the OPSS WFMS. IEEE Trans. Softw. Eng. 27, 9, 827-850.

DiAs, D. M., Kish, W., MukherJee, R., AND Tewari, R. 1996. A scalable and highly available web server. In Proceedings of the IEEE International Computer Conference (COMPCON'96). IEEE Computer Society, Los Alamitos, CA, 85-92.

Fabret, F., Jacobsen, H. A., Llirbat, F., Pereira, J., Ross, K. A., And Shasha, D. 2001. Filtering algorithms and implementation for very fast publish/subscribe systems. In Proceedings of the ACM SIGMOD International Conference on Management of Data (SIGMOD'01). ACM, New York, 115-126.

FAWCETt, T. AND Provost, F. 1999. Activity monitoring: Noticing interesting changes in behavior. In Proceedings of the ACM SIGKDD International Conference on Knowledge Discovery and Data Mining (KDD'99). ACM, New York, 53-62.

Fidler, E., Jacobsen, H. A., Li, G., And Mankovski, S. 2005. The PADRES distributed publish/subscribe system. In Proceedings of the International Conference on Feature Interactions in Telecommunications and Software Systems. 12-30.

GS1. 2008. http://www.gs1.org/docs/gdsn/gdsn_brochure.pdf.

Gupta, A., Sahin, O. D., Agrawal, D., And Abbadi, A. E. 2004. Meghdoot: Content-based publish/subscribe over P2P networks. In Proceedings of the ACM /IFIP/USENIX International Conference on Middleware (Middleware’04). Springer-Verlag, Berlin, 254-273.

Ho, K. S. AND LeONG, H. V. 2000. An extended CORBA event service with support for load balancing and fault-tolerance. In Proceedings of the International Symposium on Distributed Objects and Applications (DOA'00). IEEE Computer Society, Los Alamitos, CA, 49-58.

Hou, S. AND Jacobsen, H.-A. 2006. Predicate-based filtering of XPath expressions. In Proceedings of the International Conference on Data Engineering (ICDE'06). IEEE Computer Society, Los Alamitos, CA, 53-59.

ACM Transactions on Computer Systems, Vol. 28, No. 4, Article 9, Pub. date: December 2010. 
Hu, S., Muthusamy, V., Li, G., And Jacobsen, H.-A. 2008. Distributed automatic service composition in large-scale systems. In Proceedings of the International Conference on Distributed Event-based Systems (DEBS'08). ACM, New York, 233-244.

IBM. 2008. Service Oriented Architecture (SOA). http://www-306.ibm.com/software/solutions/soa/.

IBM, BEA Systems, Microsoft, SAP AL, ANd Siebel Systems. 2007. Business process execution language for web services version 1.1. http://www.ibm.com/developerworks/library/specification/ws-bpel/.

Jul, E., Levy, H., Hutchinson, N., AND Black, A. 1988. Fine-grained mobility in the emerald system. ACM Trans. Comput. Syst. 6, 1, 109-133.

LI, G. AND JACOBSEn, H.-A. 2005. Composite subscriptions in content-based publish/subscribe systems. In Proceedings of the ACM/IFIP/USENIX 2005 International Conference on Middleware (Middleware'05). Springer-Verlag, Berlin, 249-269.

Li, G., Cheung, A., Hou, S., Hu, S., Muthusamy, V., Sherafat, R., Wun, A., Jacobsen, H.-A., AND MANOVsKI, S. 2007. Historic data access in publish/subscribe. In Proceedings of the Inaugural International Conference on Distributed Event-Based Systems (DEBS'07). ACM, New York, 80-84.

LI, G., Hou, S., AND JACOBSEn, H.-A. 2008a. Routing of XML and XPath queries in data dissemination networks. In Proceedings of the International Conference on Distributed Computing Systems (ICDCS'08). IEEE Computer Society, Los Alamitos, CA, 627-638.

Li, G., Muthusamy, V., And Jacobsen, H.-A. 2008b. Adaptive content-based routing in general overlay topologies. In Proceedings of the ACM/IFIP/USENIX International Conference on Middleware (Middleware'08). Springer-Verlag, Berlin, 1-21.

Li, G., Muthusamy, V., And Jacobsen, H.-A. 2010. A distributed service-oriented architecture for business process execution. ACM Trans. Web 4, 1, 1-33.

LiNDERMEIER, M. 2000. Load management for distributed object-oriented environments. In Proceedings of the International Symposium on Distributed Objects and Applications (DOA'00). IEEE Computer Society, Los Alamitos, CA, 59-68.

Litzkow, M. J. 1987. Remote Unix: Turning idle workstations into cycle servers. In Summer USENIX Conference Proceedings.

Microsoft. 2008. Service Oriented Architecture. http://msdn.microsoft.com/en-us/architecture/aa948857.aspx.

MSRG. 2008. Padres project. http://msrg.org/projects/padres/.

MÜHL, G. 2001. Generic constraints for content-based publish/subscribe. In Proceedings of the International Conference on Cooperative Information Systems (CooplS'01). Springer-Verlag, Berlin, $211-225$.

MukherJee, B., Heberlein, L. T., ANd Levitt, K. N. 1994. Network intrusion detection. IEEE Netw. 8, 3, 26-41.

Muthusamy, V., Petrovic, M., And Jacobsen, H.-A. 2005. Effects of routing computations in content-based routing networks with mobile data sources. In Proceedings of the Annual International Conference on Mobile Computing and Networking (MobiCom'05). ACM, New York, 103-116.

Muthusamy, V., Jacobsen, H.-A., Coulthard, P., Chan, A., Waterhouse, J., And Litani, E. 2007. SLA-driven business process management in SOA. In Proceedings of the 2007 Conference of the Center for Advanced Studies on Collaborative Research (CASCON'07). ACM, New York, 264-267.

Oki, B., Pfluegl, M., Siegel, A., And Skeen, D. 1993. The information bus: An architecture for extensible distributed systems. In Proceedings of the ACM Symposium on Operating Systems Principles (SOSP'93). ACM, New York, 58-68.

Opyrchal, L., Astley, M., Auerbach, J., Banavar, G., Strom, R., And Sturman, D. 2000. Exploiting IP multicast in content-based publish-subscribe systems. In Proceedings of the ACM/IFIP International Conference on Distributed Systems Platforms (Middleware'00). Springer-Verlag, Berlin, 185-207. 
Othman, O., Balasubramanian, J., And Schmidt, D. C. 2003. The design of an adaptive middleware load balancing and monitoring service. In Proceedings of the International Workshop on Self-Adaptive Software (IWSAS'03). ACM, New York, 205-213.

PAllickara, S. AND Fox, G. 2003. Naradabrokering: A distributed middleware framework and architecture for enabling durable peer-to-peer grids. In Proceedings of the ACM / IFIP/USENIX International Conference on Middleware (Middleware'03). Springer-Verlag, Berlin, 41-61.

Patel, J. A., Rivière, E., Gupta, I., AND Kermarrec, A.-M. 2009. Rappel: Exploiting interest and network locality to improve fairness in publish-subscribe systems. Comput. Netw. 53, 13, $2304-2320$.

Pereira, J., Fabret, F., Jacobsen, H.-A., Llirbat, F., and Shasha, D. 2001. WebFilter: A high-throughput XML-based publish and subscribe system. In Proceedings of the International Conference on Very Large Data Bases (VLDB'01). Morgan Kaufmann, San Francisco, CA, 723-724.

Petrovic, M., Liu, H., And Jacobsen, H.-A. 2005. G-ToPSS: Fast filtering of graph-based metadata. In Proceedings of the International Conference on World Wide Web (WWW'05). ACM, New York, 539-547.

Pietzuch, P. R. AND BACON, J. 2002. Hermes: A distributed event-based middleware architecture. In Proceedings of the International Conference on Distributed Computing Systems (ICDCSW'02). IEEE Computer Society, Los Alamitos, CA, 611-618.

Reumann, J. 2009. Pub/sub at Google. CANOE and EuroSys Summer School.

Riabov, A., LiU, Z., Wolf, J. L., Yu, P. S., AND Zhang, L. 2002. Clustering algorithms for content-based publication-subscription systems. In Proceedings of the International Conference on Distributed Computing Systems (ICDCS'02). IEEE Computer Society, Los Alamitos, CA, 133-142.

Riabov, A., LiU, Z., Wolf, J. L., Yu, P. S., AND Zhang, L. 2003. New algorithms for contentbased publication-subscription systems. In Proceedings of the International Conference on Distributed Computing Systems (ICDCS'03). IEEE Computer Society, Los Alamitos, CA, 678-686.

Rose, I., Murty, R., Pietzuch, P., Ledlie, J., Roussopoulos, M., And Welsh, M. 2007. Cobra: Content-based filtering and aggregation of blogs and RSS feeds. In Proceedings of the USENIX Symposium on Networked Systems Design \& Implementation (NSDI'07).

Sachs, K., Kounev, S., Bacon, J., And Buchmann, A. 2009. Performance evaluation of message-oriented middleware using the SPECjms2007 benchmark. Perform. Eval. 66, 8, 410-434.

SChuler, C., SCHUldT, H., AND SCHEK, H.-J. 2001. Supporting reliable transactional business processes by publish/subscribe techniques. In Proceedings of the 2nd International Workshop on Technologies for E-Services (TES'01). Springer-Verlag, Berlin, 118-131.

SEgAll, B. AND ARNold, D. 1997. Elvin has left the building: A publish/subscribe notification service with quenching. In Proceedings of the Australian UNIX and Open Systems User Group Conference (AUUG'97).

SHIRRIFF, K. 1997. Building distributed process management on an object-oriented framework. In Proceedings of the Annual Conference on USENIX Annual Technical Conference (ATEC'97). USENIX Association, Berkeley, CA, 9-22.

TAM, D., Azimi, R., AND JACOBSEn, H.-A. 2003. Building content-based publish/subscribe systems with distributed hash tables. In Proceedings of the International Workshop on Databases, Information Systems and Peer-to-Peer Computing. 138-152.

Tatbul, N., Çetintemel, U., AND Zdonik, S. 2007. Staying FIT: Efficient load shedding techniques for distributed stream processing. In Proceedings of the International Conference on Very Large Data Bases (VLDB'07). VLDB Endowment, 159-170.

Terpstra, W. W., Behnel, S., Fiege, L., Zeidler, A., And Buchmann, A. P. 2003. A peer-topeer approach to content-based publish/subscribe. In Proceedings of the International Workshop on Distributed Event-Based Systems (DEBS'03). ACM, New York, 1-8.

TIBCO. 2001. TIBCO software chosen as infrastructure for NASDAQ's supermontage. www.tibco.com.

ACM Transactions on Computer Systems, Vol. 28, No. 4, Article 9, Pub. date: December 2010. 
TRiantafillou, P. AND AEKATERINidis, I. 2004. Content-based publish/subscribe systems over structured P2P networks. In Proceedings of the International Workshop on Distributed Event Based Systems (DEBS'04).

VITRIA. 2008. http://www.vitria.com/.

Voulgaris, S., Rivire, E., Kermarrec, A.-M., And Steen, M. V. 2006. Sub-2-Sub: Selforganizing content-based publish subscribe for dynamic large scale collaborative networks. In Proceedings of the International Workshop on Peer-to-Peer Systems (IPTPS06).

Wong, T., KATZ, R. H., AND MCCANnE, S. 2000. An evaluation of preference clustering in largescale multicast applications. In Proceedings of the IEEE International Conference on Computer Communications (INFOCOM'00). 451-460.

Yan, W., Hu, S., Muthusamy, V., Jacobsen, H.-A., AND ZHA, L. 2009. Efficient event-based resource discovery. In Proceedings of the ACM International Conference on Distributed EventBased Systems (DEBS'09). ACM, New York, 1-12.

Zajcew, R., Roy, P., Black, D., Peak, C., Guedes, P., Kemp, B., LoVerso, J., Leibensperger, M., Barnett, M., Rabit, F., ANd Netterwala, D. 1993. An OSF/1 UNIX for massively parallel multicomputers. In Proceedings of the Winter USENIX Conference. 37-55.

Zhang, C., Krishnamurthy, A., WANG, R. Y., AND Singh, J. P. 2005. Combining flexibility and scalability in a peer-to-peer publish/subscribe system. In Proceedings of the ACM / IFIP/USENIX 2005 International Conference on Middleware (Middleware’05). Springer-Verlag, Berlin, $102-123$.

ZHU, Y. AND HU, Y. 2007. Ferry: A P2P-based architecture for content-based publish/subscribe services. IEEE Trans. Parall. Distrib. Syst. 18, 5, 672-685.

Received August 2008; revised March 2010; accepted August 2010 